Groups Geom. Dyn. 6 (2012), 765-801

DOI $10.4171 / \mathrm{GGD} / 174$
Groups, Geometry, and Dynamics

(C) European Mathematical Society

\title{
Existence, covolumes and infinite generation of lattices for Davis complexes
}

\author{
Anne Thomas*
}

\begin{abstract}
Let $\Sigma$ be the Davis complex for a Coxeter system $(W, S)$. The automorphism group $G$ of $\Sigma$ is naturally a locally compact group, and a simple combinatorial condition due to Haglund-Paulin and White determines when $G$ is nondiscrete. The Coxeter group $W$ may be regarded as a uniform lattice in $G$. We show that many such $G$ also admit a nonuniform lattice $\Gamma$, and an infinite family of uniform lattices with covolumes converging to that of $\Gamma$. It follows that the set of covolumes of lattices in $G$ is nondiscrete. We also show that the nonuniform lattice $\Gamma$ is not finitely generated. Examples of $\Sigma$ to which our results apply include buildings and non-buildings, and many complexes of dimension greater than 2 . To prove these results, we introduce a new tool, that of "group actions on complexes of groups", and use this to construct our lattices as fundamental groups of complexes of groups with universal cover $\Sigma$.
\end{abstract}

Mathematics Subject Classification (2010). 22D05, 22F50, 20E42, 57M07.

Keywords. Lattice, Davis complex, Coxeter group, building, complex of groups.

\section{Introduction}

Let $G$ be a locally compact topological group, with Haar measure $\mu$. A discrete subgroup $\Gamma \leq G$ is a lattice if $\Gamma \backslash G$ carries a finite $G$-invariant measure, and is uniform if $\Gamma \backslash G$ is compact. Some basic questions are:

(1) Does $G$ admit a (uniform or nonuniform) lattice?

(2) What is the set of covolumes of lattices in $G$, that is, the set of positive reals

$$
\mathcal{V}(G):=\{\mu(\Gamma \backslash G) \mid \Gamma<G \text { is a lattice }\} \text { ? }
$$

(3) Are lattices in $G$ finitely generated?

These questions have been well studied in classical cases. For example, suppose that $G$ is a reductive algebraic group over a local field $K$ of characteristic 0 . Then $G$ admits a uniform lattice, constructed by arithmetic means (Borel-Harder [4]), and a

\footnotetext{
* This work was supported in part by NSF Grant No. DMS-0805206 and in part by EPSRC Grant No. EP/D073626/2. The author is currently supported by ARC Grant No. DP110100440.
} 
nonuniform lattice only if $K$ is archimedean (Tamagawa [28]). If $G$ is a semisimple real Lie group, the set $\mathcal{V}(G)$ is in most cases discrete (see [23] and its references). If in addition $G$ is simple and higher-rank, then $G$ and hence its lattices have Kazhdan's property (T) (see, for example, [24]). Since countable groups with property (T) are finitely generated, it follows that all lattices in $G$ are finitely generated.

A nonclassical case is $G$ the automorphism group of a locally finite tree $T$. The study of lattices in $G=\operatorname{Aut}(T)$ was initiated by Bass and Lubotzky, and has yielded many surprising differences from classical results (see the survey [23] and the reference [3]). For example, the set $\mathcal{V}(G)$ is in many cases nondiscrete, and nonuniform tree lattices are never finitely generated.

In fact, the automorphism group $G$ of any locally finite polyhedral complex $X$ is naturally a locally compact group (see Section 2.1). For many $X$ with $\operatorname{dim}(X) \geq 2$, there is greater rigidity than for trees, as might be expected in higher dimensions. For instance, Burger-Mozes [9] proved a 'Normal Subgroup Theorem' for products of trees (parallel to that of Margulis [24] for higher-rank semisimple Lie groups), and Bourdon-Pajot [7] and Xie [33] established quasi-isometric rigidity for certain Fuchsian buildings. On the other hand, lattices in $G=\operatorname{Aut}(X)$ can exhibit the same flexibility as tree lattices. For example, the set $\mathcal{V}(G)$ is nondiscrete for certain right-angled buildings [29] and Fuchsian buildings [30]. Another example is density of commensurators of uniform lattices in $G$, proved by Haglund [15] for certain 2dimensional Davis complexes, and by Haglund [18] and Kubena-Thomas [21] for right-angled buildings. Apart from right-angled buildings, very little is known for $X$ of dimension $>2$. Almost nothing is known for $X$ not a building.

In this paper we consider Questions (1)-(3) above for lattices in $G=\operatorname{Aut}(\Sigma)$, where $\Sigma$ is the Davis complex for a Coxeter system $(W, S)$ (see [11] and Section 2.2 below). The Davis complex is a locally finite, piecewise Euclidean CAT( 0$)$ polyhedral complex, and the Coxeter group $W$ may be regarded as a uniform lattice in $G$. Our results are the Main Theorem and its Corollaries 1.1 and 1.2 below, which establish tree-like properties for lattices in many such $G$. After stating these results, we discuss how they apply to (barycentric subdivisions of) Fuchsian buildings and Platonic polygonal complexes, and to many Davis complexes $\Sigma$ with $\operatorname{dim}(\Sigma)>2$.

To state the Main Theorem, recall that for a Coxeter system $(W, S)$ with $W=\langle S|$ $\left.(s t)^{m_{s t}}\right\rangle$, and any $T \subset S$, the special subgroup $W_{T}$ is the subgroup of $W$ generated by the elements $s \in T$. A special subgroup $W_{T}$ is spherical if it is finite, and the set of spherical special subgroups of $W$ is partially ordered by inclusion. The poset of nontrivial spherical special subgroups is an abstract simplicial complex $L$, called the nerve of $(W, S)$. We identify each generator $s \in S$ with the corresponding vertex $W_{\{s\}}=\langle s\rangle$ of $L$, and denote by $A$ the group of label-preserving automorphisms of $L$, that is, the group of automorphisms $\alpha$ of $L$ such that $m_{s t}=m_{\alpha(s) \alpha(t)}$ for all $s, t \in S$. The group $G=\operatorname{Aut}(\Sigma)$ is nondiscrete if and only if there is a nontrivial $\alpha \in A$ such that $\alpha$ fixes the star in $L$ of some vertex $s$ (see Haglund-Paulin [19] and also White [32]). 
Main Theorem. Let $(W, S)$ be a Coxeter system, with nerve L and Davis complex $\Sigma$. Let $A$ be the group of label-preserving automorphisms of L. Assume that there are vertices $s_{1}$ and $s_{2}$ of $L$, and nontrivial elements $\alpha_{1}, \alpha_{2} \in A$ such that for $i=1,2$ :

(1) $\alpha_{i}$ fixes the star of $s_{3-i}$ in $L$;

(2) the subgroup $\left\langle\alpha_{i}\right\rangle$ of $A$ acts freely on the $\left\langle\alpha_{i}\right\rangle$-orbit of $s_{i}$, in particular $\alpha_{i}\left(s_{i}\right) \neq s_{i}$;

(3) for all $t_{i} \neq s_{i}$ such that $t_{i}$ is in the $\left\langle\alpha_{i}\right\rangle$-orbit of $s_{i}, m_{s_{i}} t_{i}=\infty$; and

(4) all spherical special subgroups $W_{T}$ with $s_{i} \in T$ are halvable along $s_{i}$ (see Definition 1.4 below).

Then $G=\operatorname{Aut}(\Sigma)$ admits

- a nonuniform lattice $\Gamma$; and

- an infinite family of uniform lattices $\left(\Gamma_{n}\right)$ such that $\mu\left(\Gamma_{n} \backslash G\right) \rightarrow \mu(\Gamma \backslash G)$, where $\mu$ is Haar measure on $G$.

Corollary 1.1. The set of covolumes of lattices in $G$ is nondiscrete.

Corollary 1.2. The group $G$ admits a lattice which is not finitely generated.

Corollary 1.2 follows from the proof of the Main Theorem and Theorem 1.3 below. By the discussion above, Corollary 1.2 implies that the group $G$ in the Main Theorem does not have property (T). This was already known for $G=\operatorname{Aut}(\Sigma)$, where $\Sigma$ is any Davis complex (Haglund-Paulin [19]); our results thus provide an alternative proof of this fact in some cases.

We describe several infinite families of examples of Davis complexes $\Sigma$ to which our results apply in Section 5 below. To establish these applications, we use properties of spherical buildings in [25], and some results of graph theory from [12]. In two dimensions, examples include the Fuchsian buildings considered in [30], and some of the highly symmetric Platonic polygonal complexes investigated by Świątkowski [27]. Platonic polygonal complexes are not in general buildings, and even the existence of lattices (other than the Coxeter group $W$ ) in their automorphism groups was not previously known. An example of a Platonic polygonal complex is the (unique) CAT(0) 2-complex with all 2-cells squares, and the link of every vertex the Petersen graph (Figure 1 below). The Main Theorem and its corollaries also apply to many higher-dimensional $\Sigma$, including both buildings and complexes which are not buildings.

To prove the Main Theorem, we construct the lattices $\Gamma_{n}$ and $\Gamma$ as fundamental groups of complexes of groups with universal covers $\Sigma$ (see [8] and Section 2.3 below). The construction is given in Section 4 below, where we also prove Corollary 1.2.

Complexes of groups are a generalisation to higher dimensions of graphs of groups. Briefly, given a polyhedral complex $Y$, a (simple) complex of groups $G(Y)$ over $Y$ is an assignment of a local group $G_{\sigma}$ to each cell $\sigma$ of $Y$, with monomorphisms $G_{\sigma} \rightarrow G_{\tau}$ whenever $\tau \subset \sigma$, so that the obvious diagrams commute. The action of a group $G$ on a polyhedral complex $X$ induces a complex of groups $G(Y)$ 


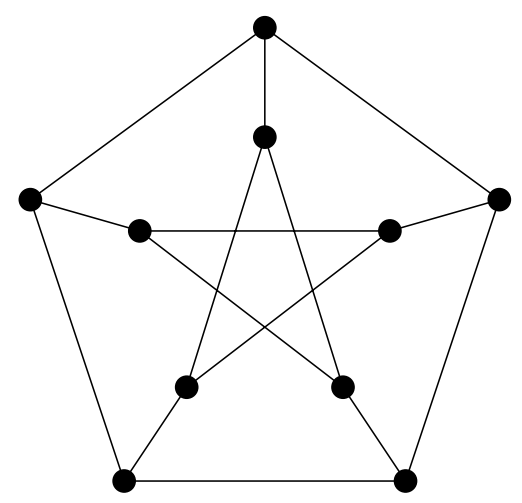

Figure 1. Petersen graph.

over $Y=G \backslash X$. A complex of groups is developable if it is isomorphic to a complex of groups induced in this way. A developable complex of groups $G(Y)$ has a simply-connected universal cover $\widehat{G(Y)}$, equipped with a canonical action of the fundamental group of the complex of groups $\pi_{1}(G(Y))$.

A key difference from graphs of groups is that complexes of groups are not in general developable. In addition, even if $G(Y)$ is developable, with universal cover say $X$, it may be impossible to identify $X$ of dimension $\geq 2$ using only local data such as the links of its vertices (see Ballmann-Brin [1] and Haglund [14]). To ensure that our complexes of groups are developable with universal cover $\Sigma$, we use covering theory for complexes of groups (see [8] and [22], and Section 3.1 below). The main result needed is that if there is a covering of complexes of groups $G(Y) \rightarrow H(Z)$, then $G(Y)$ is developable if and only if $H(Z)$ is developable, and the universal covers of $G(Y)$ and $H(Z)$ are isometric (see Theorem 3.2 below).

The other main ingredient in the proof of the Main Theorem is Theorem 1.3 below, which introduces a theory of "group actions on complexes of groups". This is a method of manufacturing new complexes of groups with a given universal cover, by acting on previously-constructed complexes of groups. Given a complex of groups $G(Y)$, and the action of a group $H$ on $Y$, the $H$-action extends to an action on $G(Y)$ if there is a homomorphism from $H$ to $\operatorname{Aut}(G(Y))$. Roughly, this means that for each cell $\sigma$ of $Y$, each $h \in H$ induces a group isomorphism $G_{\sigma} \rightarrow G_{h \cdot \sigma}$, so that the obvious diagrams commute (see Section 3.1 below for definitions). In Section 3 below we prove:

Theorem 1.3. Let $G(Y)$ be a (simple) complex of groups over $Y$, and suppose that the action of a group $H$ on $Y$ extends to an action on $G(Y)$. Then the $H$-action induces a complex of groups $H(Z)$ over $Z=H \backslash Y$ such that there is a covering of complexes of groups $G(Y) \rightarrow H(Z)$. Moreover there is a natural short exact 
sequence

$$
1 \rightarrow \pi_{1}(G(Y)) \rightarrow \pi_{1}(H(Z)) \rightarrow H \rightarrow 1,
$$

and if $H$ fixes a vertex of $Y$, then

$$
\pi_{1}(H(Z)) \cong \pi_{1}(G(Y)) \rtimes H .
$$

Theorem 1.3 is also used in [21], and we expect this result to be of independent interest. To our knowledge, group actions on complexes of groups have not previously been considered. In [2], Bass-Jiang determined the structure of the full automorphism group of a graph of groups, but did not define or study the graph of groups induced by a group action on a graph of groups. A more precise statement of Theorem 1.3, including some additional facts about $H(Z)$, is given as Theorem 3.1 below.

The Main Theorem is proved as follows. The action of the Coxeter group $W$ on $\Sigma$ induces a complex of groups $G\left(Y_{1}\right)$ over $Y_{1}=W \backslash \Sigma$, with local groups the spherical special subgroups of $W$. We then construct a family of finite complexes of groups $G\left(Y_{n}\right)$ and $H\left(Z_{n}\right)$, and two infinite complexes of groups $G\left(Y_{\infty}\right)$ and $H\left(Z_{\infty}\right)$, so that there are coverings of complexes of groups as sketched in Figure 2 below.
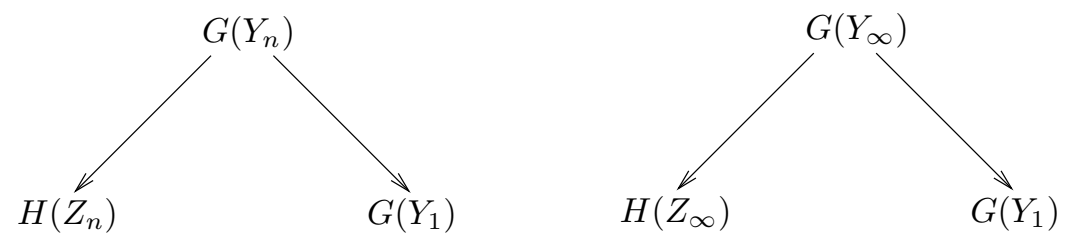

Figure 2. Coverings of complexes of groups.

The fundamental groups of $H\left(Z_{n}\right)$ and $H\left(Z_{\infty}\right)$ are, respectively, the uniform lattices $\Gamma_{n}$, and the nonuniform lattice $\Gamma$, in $G=\operatorname{Aut}(\Sigma)$. For the local groups of $G\left(Y_{n}\right)$ and $G\left(Y_{\infty}\right)$, we use condition (4) in the Main Theorem to replace certain spherical special subgroups $W_{T}$ by the subgroup half ${ }_{S}\left(W_{T}\right)$, defined as follows:

Definition 1.4. Let $W_{T}$ be a spherical special subgroup of $W$, and suppose $s \in T$. Then $W_{T}$ is halvable along $s$ if the union

$$
(T-\{s\}) \cup\{s t s \mid t \in T-\{s\}\}
$$

generates an index 2 subgroup, denoted by half ${ }_{s}\left(W_{T}\right)$, of $W_{T}$.

The complexes of groups $H\left(Z_{n}\right)$ and $H\left(Z_{\infty}\right)$ are induced by group actions on, respectively, $G\left(Y_{n}\right)$ and $G\left(Y_{\infty}\right)$. To construct these group actions, we use the automorphisms $\alpha_{1}$ and $\alpha_{2}$ of $L$.

I am grateful to Benson Farb for introducing me to these questions, and for his continuing encouragement and advice. I also thank G. Christopher Hruska and Kevin 
Wortman for many useful discussions. This particular work was inspired by conversations with Tadeusz Januszkiewicz and Jacek Świątkowski, and much of this project was carried out at the Mathematical Sciences Research Institute in Fall 2007, where I benefited from talking with Angela Kubena, Michael W. Davis, Jonathan P. McCammond and Damian Osajda. I would also like to thank Karen Vogtmann for helpful comments on this manuscript, and an anonymous referee for careful reading and worthwhile suggestions.

\section{Background}

In this section we present brief background. In Section 2.1 we describe the natural topology on $G$ the group of automorphisms of a locally finite polyhedral complex $X$, and characterise uniform and nonuniform lattices in $G$. Section 2.2 constructs the Davis complex $\Sigma$ for a Coxeter system $(W, S)$, following [11]. In Section 2.3 we recall the basics of Haefliger's theory of complexes of groups (see [8]), and describe the complex of groups $G\left(Y_{1}\right)$ induced by the action of $W$ on $\Sigma$.

2.1. Lattices in automorphism groups of polyhedral complexes. Let $G$ be a locally compact topological group. We will use the following normalisation of Haar measure $\mu$ on $G$.

Theorem 2.1 (Serre, [26]). Suppose that a locally compact group $G$ acts on a set $V$ with compact open stabilisers and a finite quotient $G \backslash V$. Then there is a normalisation of the Haar measure $\mu$ on $G$, depending only on the choice of $G$-set $V$, such that for each discrete subgroup $\Gamma$ of $G$ we have

$$
\mu(\Gamma \backslash G)=\operatorname{Vol}(\Gamma \backslash V):=\sum_{v \in \Gamma \backslash V} \frac{1}{\left|\Gamma_{v}\right|} \leq \infty .
$$

Suppose $X$ is a connected, locally finite polyhedral complex. Let $G=\operatorname{Aut}(X)$. With the compact-open topology, $G$ is naturally a locally compact topological group, and the $G$-stabilisers of cells in $X$ are compact and open. Hence if $G \backslash X$ is finite, there are several natural choices of sets $V$ in Theorem 2.1 above. By the same arguments as for tree lattices ([3], Chapter 1), it can be shown (for any suitable set $V$ ) that a discrete subgroup $\Gamma \leq G$ is a lattice if and only if the series $\operatorname{Vol}(\Gamma \backslash V)$ converges, and $\Gamma$ is uniform if and only if this is a sum with finitely many terms. In Section 2.2 below we describe our choice of $G$-set $V$ when $G$ is the group of automorphisms of a Davis complex $\Sigma$.

2.2. Davis complexes. In this section we recall the construction of the Davis complex for a Coxeter system. We follow the reference [11]. 
A Coxeter group is a group $W$ with a finite generating set $S$ and presentation of the form

$$
\left.W=\langle s \in S| s^{2}=1 \text { for all } s \in S,(s t)^{m_{s t}}=1 \text { for all } s, t \in S \text { with } s \neq t\right\rangle
$$

with $m_{s t}$ an integer $\geq 2$ or $m_{s t}=\infty$, meaning that $s t$ has infinite order. The pair $(W, S)$ is called a Coxeter system.

Example 1. This example will be followed throughout this section, and also referred to in Sections 2.3 and 4 below. Let

$$
\begin{gathered}
W=\left\langle s_{1}, s_{2}, s_{3}, s_{4}, s_{5}\right| s_{i}^{2}=1,\left(s_{1} s_{4}\right)^{m}=\left(s_{2} s_{4}\right)^{m}=\left(s_{3} s_{4}\right)^{m}=1, \\
\left.\left(s_{1} s_{5}\right)^{m^{\prime}}=\left(s_{2} s_{5}\right)^{m^{\prime}}=\left(s_{3} s_{5}\right)^{m^{\prime}}=1\right\rangle
\end{gathered}
$$

where $m$ and $m^{\prime}$ are integers $\geq 2$.

Let $(W, S)$ be a Coxeter system. A subset $T$ of $S$ is spherical if the corresponding special subgroup $W_{T}$ is spherical, that is, $W_{T}$ is finite. By convention, $W_{\emptyset}$ is the trivial group. Denote by $S$ the set of all spherical subsets of $S$. The set $S$ is partially ordered by inclusion, and the poset $S_{>\emptyset}$ is the nerve $L$ of $(W, S)$ (this is equivalent to the description of $L$ in the introduction above). By definition, a nonempty set $T$ of vertices of $L$ spans a simplex $\sigma_{T}$ in $L$ if and only if $T$ is spherical. We identify the generator $s \in S$ with the vertex $\{s\}$ of $L$. The vertices $s$ and $t$ of $L$ are joined by an edge in $L$ if and only if $m_{s t}$ is finite, in which case we label this edge by the integer $m_{s t}$. The nerve $L$ of Example 1 above, with its edge labels, is sketched in Figure 3 below.

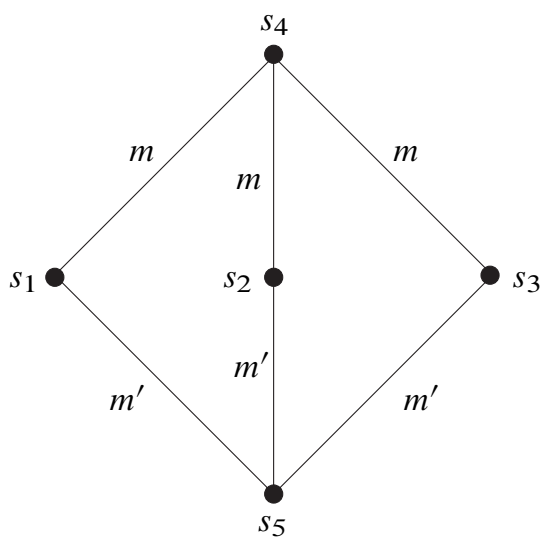

Figure 3. Nerve $L$ of Example 1, with edge labels.

We denote by $K$ the geometric realisation of the poset $S$. Equivalently, $K$ is the cone on the barycentric subdivision of the nerve $L$ of $(W, S)$. Note that $K$ is compact 
and contractible, since it is the cone on a finite simplicial complex. Each vertex of $K$ has type a spherical subset of $S$, with the cone point having type $\emptyset$. For Example 1 above, $K$ and the types of its vertices are sketched on the left of Figure 4.
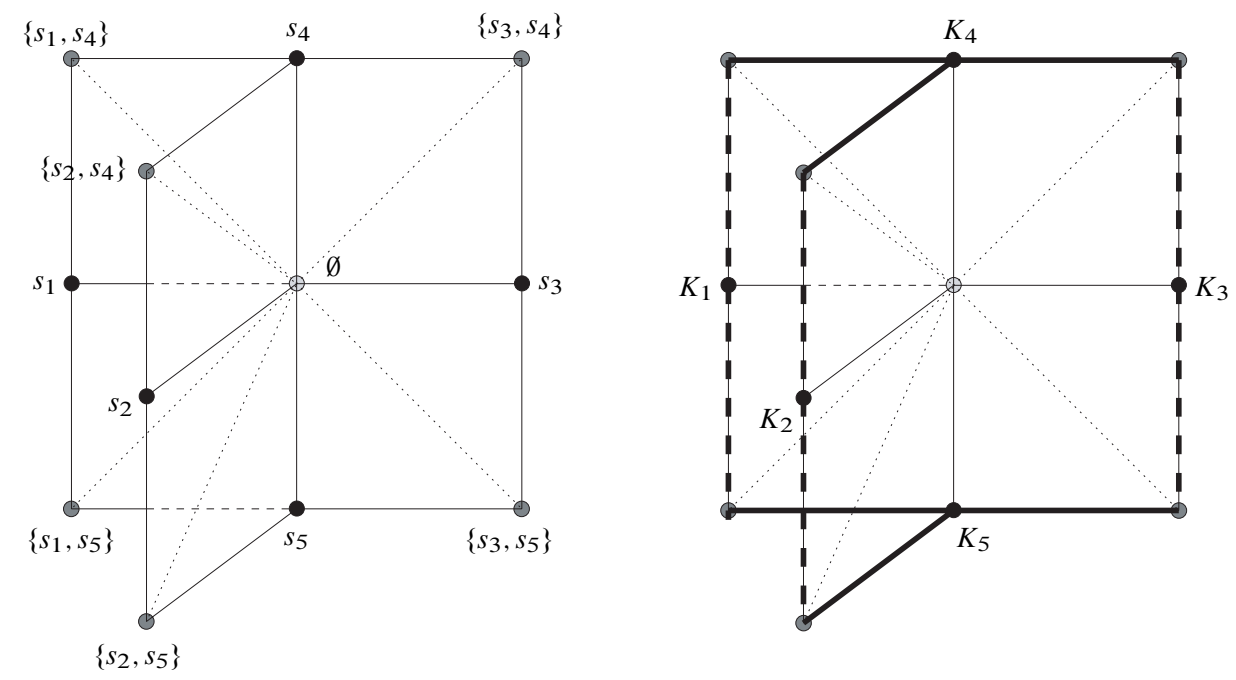

Figure 4. $K$ and types of vertices (left) and mirrors (right) for Example 1.

For each $s \in S$ let $K_{s}$ be the union of the (closed) simplices in $K$ which contain the vertex $s$ but not the cone point. In other words, $K_{s}$ is the closed star of the vertex $s$ in the barycentric subdivision of $L$. Note that $K_{s}$ and $K_{t}$ intersect if and only if $m_{s t}$ is finite. The family $\left(K_{s}\right)_{s \in S}$ is a mirror structure on $K$, meaning that $\left(K_{S}\right)_{s \in S}$ is a family of closed subspaces of $K$, called mirrors. We call $K_{S}$ the s-mirror of $K$. For Example 1 above, the mirrors $K_{i}=K_{s_{i}}$ are shown in heavy lines on the right of Figure 4.

For each $x \in K$, put

$$
S(x):=\left\{s \in S \mid x \in K_{s}\right\} .
$$

Now define an equivalence relation $\sim$ on the set $W \times K$ by $(w, x) \sim\left(w^{\prime}, x^{\prime}\right)$ if and only if $x=x^{\prime}$ and $w^{-1} w^{\prime} \in W_{S(x)}$. The Davis complex $\Sigma$ for $(W, S)$ is then the quotient space

$$
\Sigma:=(W \times K) / \sim .
$$

The types of vertices of $K$ induce types of the vertices of $\Sigma$, and the natural $W$-action on $W \times K$ descends to a type-preserving action on $\Sigma$, with compact quotient $K$, so that the $W$-stabiliser of a vertex of $\Sigma$ of type $T \in S$ is a conjugate of the spherical special subgroup $W_{T}$.

We identify $K$ with the subcomplex $(1, K)$ of $\Sigma$, and write $w K$ for the translate $(w, K)$, where $w \in W$. Any $w K$ is called a chamber of $\Sigma$. The mirrors $K_{s}$ of $K$, or 
any of their translates by elements of $W$, are called the mirrors of $\Sigma$. Two distinct chambers of $\Sigma$ are $s$-adjacent if their intersection is an $s$-mirror, and are adjacent if their intersection is an $s$-mirror for some $s \in S$. Note that the chambers $w K$ and $w^{\prime} K$ are $s$-adjacent if and only if $w^{-1} w^{\prime}=s$, equivalently $w^{\prime}=w s$ and $w^{\prime} s=w$. For Example 1 above, part of the Davis complex $\Sigma$ for $(W, S)$ is shown in Figure 5. There are $2 m$ copies of $K$ glued around the vertices of types $\left\{s_{i}, s_{4}\right\}$, for $i=1,2,3$, since $W_{\left\{s_{i}, s_{4}\right\}}$ has order $2 m$. Similarly, there are $2 m^{\prime}$ copies of $K$ glued around the vertices of types $\left\{s_{i}, s_{5}\right\}$, for $i=1,2,3$.

The Davis complex $\Sigma$ may be metrised with a piecewise Euclidean structure such that $\Sigma$ is a complete CAT(0) space (Moussong, see Theorem 12.3.3 of [11]). From now on we will assume that $\Sigma$ is equipped with this metric.

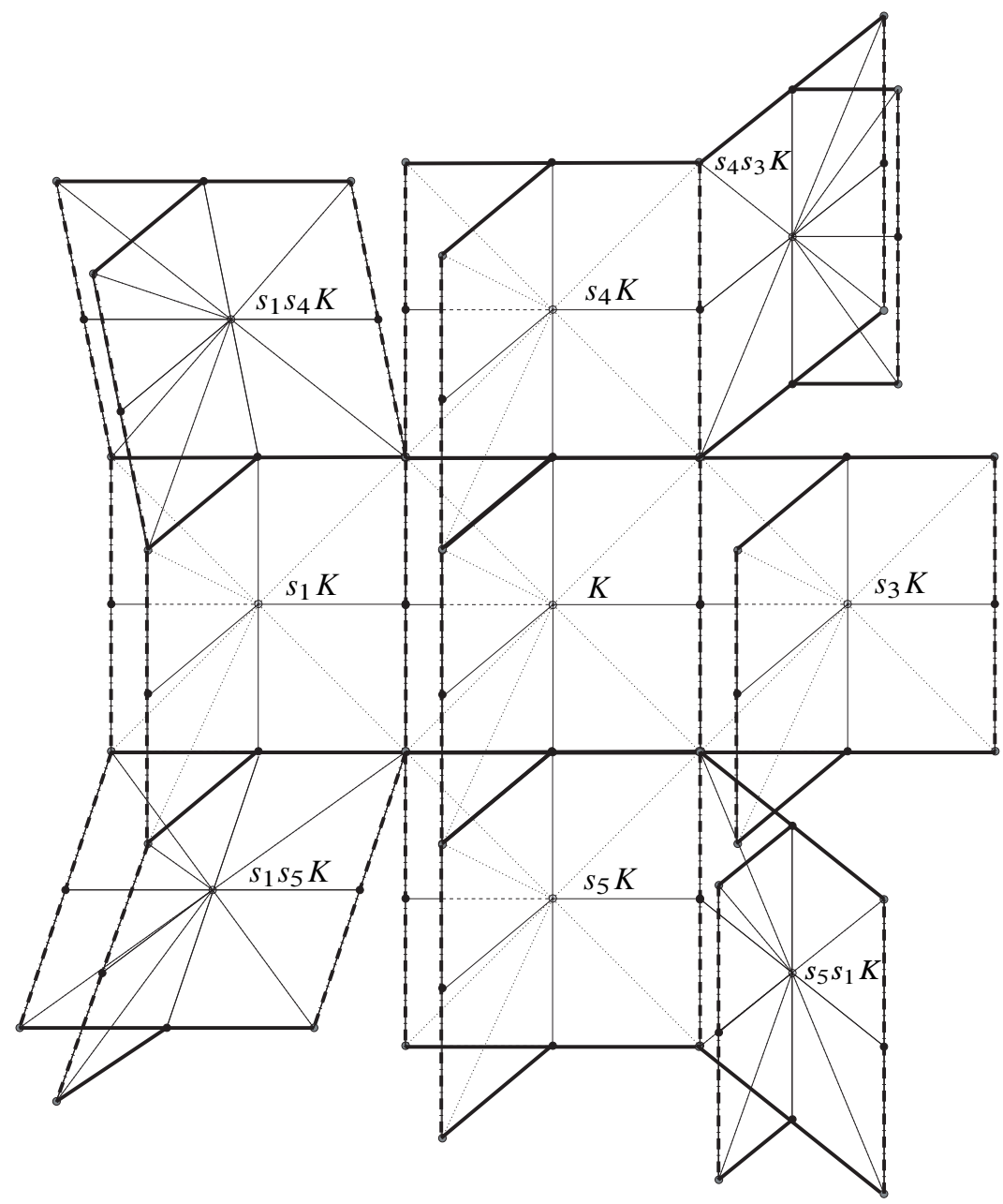

Figure 5. Part of $\Sigma$, for Example 1. 
Suppose that $G=\operatorname{Aut}(\Sigma)$ is the group of automorphisms of a Davis complex $\Sigma$. Since $W$ acts cocompactly on $\Sigma$, with finite stabilisers, it may be regarded as a uniform lattice in $G$. We take as the set $V$ in Theorem 2.1 above the set of vertices of $\Sigma$ of type $\emptyset$. Then the covolume of $W$ is 1 , since $W$ acts simply transitively on $V$.

2.3. Complexes of groups. We now outline the basic theory of complexes of groups, following Chapter III.C of [8]. The definitions of the more involved notions of morphisms and coverings of complexes of groups are postponed to Section 3.1 below.

In the literature, a complex of groups $G(Y)$ is constructed over a space or set $Y$ belonging to various different categories, including simplicial complexes, polyhedral complexes, or, most generally, scwols (small categories without loops). In each case there is a set of vertices, and a set of oriented edges with a composition law. The formal definition of a scwol is:

Definition 2.2. A scwol $X$ is the disjoint union of a set $V(X)$ of vertices and a set $E(X)$ of edges, with each edge $a$ oriented from its initial vertex $i(a)$ to its terminal vertex $t(a)$, such that $i(a) \neq t(a)$. A pair of edges $(a, b)$ is composable if $i(a)=t(b)$, in which case there is a third edge $a b$, called the composition of $a$ and $b$, such that $i(a b)=i(b), t(a b)=t(a)$ and if $i(a)=t(b)$ and $i(b)=t(c)$ then $(a b) c=a(b c)$ (associativity).

We will always assume that scwols are connected (see Section III.e.1.1 of [8]). Morphisms of scwols and group actions on scwols are defined as follows:

Definition 2.3. Let $X, Y$ and $Z$ be scwols. A nondegenerate morphism $f: Y \rightarrow Z$ is a map that sends $V(Y)$ to $V(Z)$, sends $E(Y)$ to $E(Z)$ and is such that:

(1) for each $a \in E(Y)$, we have $i(f(a))=f(i(a))$ and $t(f(a))=f(t(a))$;

(2) for each pair of composable edges $(a, b)$ in $Y$, we have $f(a b)=f(a) f(b)$; and

(3) for each vertex $\sigma \in V(Y)$, the restriction of $f$ to the set of edges with initial vertex $\sigma$ is a bijection onto the set of edges of $Z$ with initial vertex $f(\sigma)$.

A morphism of scwols $f: Y \rightarrow Z$ is a functor from the category $Y$ to the category $Z$ (see Section III. C.A.1 of [8]). An automorphism of a scwol $X$ is a morphism from $X$ to $X$ that has an inverse.

Definition 2.4. An action of a group $G$ on a scwol $X$ is a homomorphism from $G$ to the group of automorphisms of $X$ such that for all $a \in E(X)$ and all $g \in G$ :

(1) $g \cdot i(a) \neq t(a)$; and

(2) if $g \cdot i(a)=i(a)$ then $g \cdot a=a$.

Suppose now that $\Sigma$ is the Davis complex for a Coxeter system $(W, S)$, as defined in Section 2.2 above. Recall that each vertex $\sigma \in V(\Sigma)$ has type $T$ a spherical subset 
of $S$. The edges $E(\Sigma)$ are then naturally oriented by inclusion of type. That is, if the edge $a$ joins the vertex $\sigma$ of type $T$ to the vertex $\sigma^{\prime}$ of type $T^{\prime}$, then $i(a)=\sigma$ and $t(a)=\sigma^{\prime}$ exactly when $T \subsetneq T^{\prime}$. It is clear that the sets $V(\Sigma)$ and $E(\Sigma)$ satisfy the properties of a scwol. Moreover, if $Y$ is a subcomplex of $\Sigma$, then the sets $V(Y)$ and $E(Y)$ also satisfy Definition 2.2 above. By abuse of notation, we identify $\Sigma$ and $Y$ with the associated scwols.

We now define complexes of groups over scwols.

Definition 2.5. A complex of groups $G(Y)=\left(G_{\sigma}, \psi_{a}, g_{a, b}\right)$ over a scwol $Y$ is given by:

(1) a group $G_{\sigma}$ for each $\sigma \in V(Y)$, called the local group at $\sigma$;

(2) a monomorphism $\psi_{a}: G_{i(a)} \rightarrow G_{t(a)}$ along the edge $a$ for each $a \in E(Y)$; and

(3) for each pair of composable edges, a twisting element $g_{a, b} \in G_{t(a)}$, such that

$$
\operatorname{Ad}\left(g_{a, b}\right) \circ \psi_{a b}=\psi_{a} \circ \psi_{b}
$$

where $\operatorname{Ad}\left(g_{a, b}\right)$ is conjugation by $g_{a, b}$ in $G_{t(a)}$, and for each triple of composable edges $a, b, c$ the following cocycle condition holds

$$
\psi_{a}\left(g_{b, c}\right) g_{a, b c}=g_{a, b} g_{a b, c} .
$$

A complex of groups is simple if each $g_{a, b}$ is trivial.

Example. This example will be followed throughout this section, and used in the proof of the Main Theorem in Section 4 below. Let $(W, S)$ be a Coxeter system with nerve $L$ and let $K$ be the cone on the barycentric subdivision of $L$, as in Section 2.2 above. Put $Y_{1}=K$, with the orientations on edges discussed above. We construct a simple complex of groups $G\left(Y_{1}\right)$ over $Y_{1}$ as follows. Let $\sigma \in V\left(Y_{1}\right)$. Then $\sigma$ has type a spherical subset $T$ of $S$, and we define $G_{\sigma}=W_{T}$. All monomorphisms along edges of $Y_{1}$ are then natural inclusions, and all $g_{a, b}$ are trivial. For $(W, S)$ as in Example 1 of Section 2.2 above, the complex of groups $G\left(Y_{1}\right)$ is shown in Figure 6 below. In this figure, $D_{2 m}$ and $D_{2 m^{\prime}}$ are the dihedral groups of orders $2 m$ and $2 m^{\prime}$ respectively, and $C_{2}$ is the cyclic group of order 2 .

Suppose a group $G$ acts on a scwol $X$, as in Definition 2.4 above. Then the quotient $Y=G \backslash X$ also has the structure of a scwol, and the action of $G$ induces a complex of groups $G(Y)$ over $Y$ (this construction is generalised in Section 3.2 below). Let $Y=G \backslash X$ with $p: X \rightarrow Y$ the natural projection. For each $\sigma \in V(Y)$, choose a lift $\bar{\sigma} \in V(X)$ such that $p(\bar{\sigma})=\sigma$. The local group $G_{\sigma}$ of $G(Y)$ is then defined to be the stabiliser of $\bar{\sigma}$ in $G$, and the monomorphisms $\psi_{a}$ and elements $g_{a, b}$ are defined using further choices. The resulting complex of groups $G(Y)$ is unique up to isomorphism (see Definition 3.3 below).

A complex of groups is developable if it is isomorphic to a complex of groups $G(Y)$ induced, as just described, by such an action. Complexes of groups, unlike 


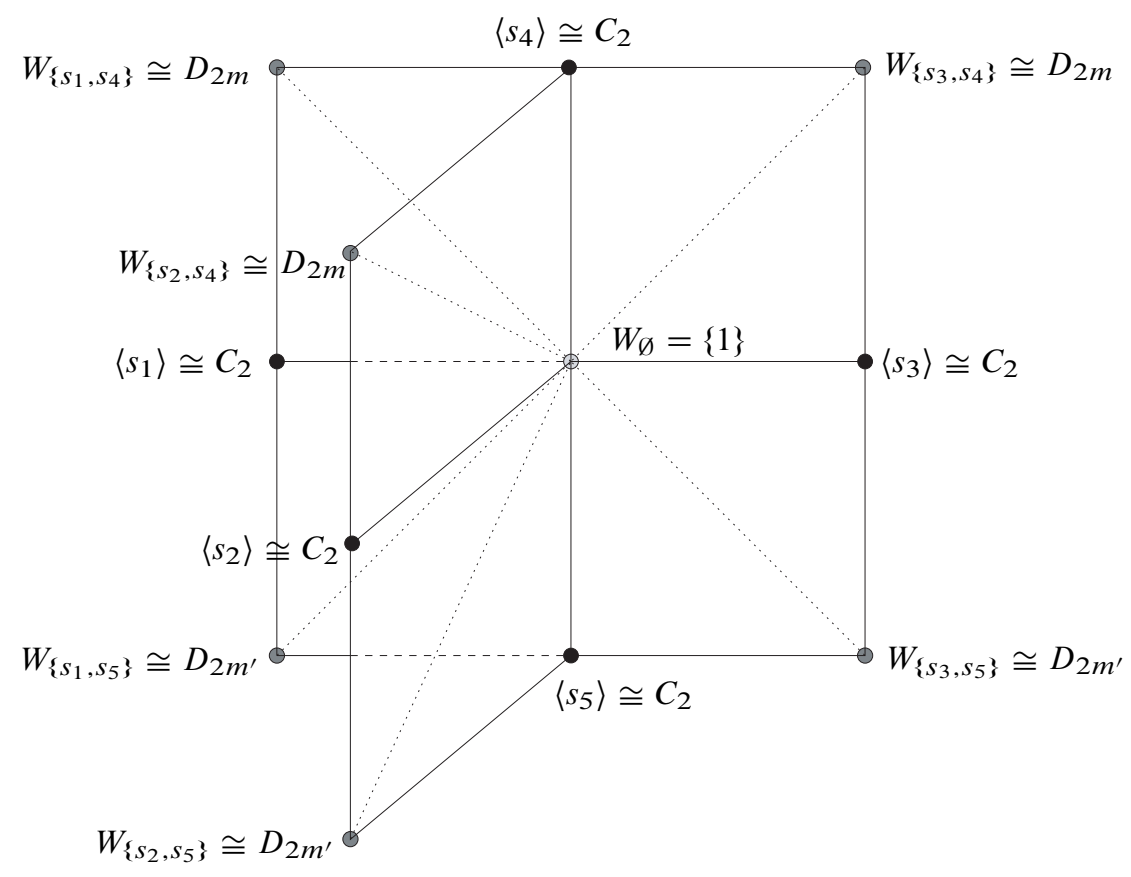

Figure 6. The complex of groups $G\left(Y_{1}\right)$, for Example 1 of Section 2.2.

graphs of groups, are not in general developable. The complex of groups $G\left(Y_{1}\right)$ above is developable, since it is the complex of groups induced by the action of $W$ on $\Sigma$, where for each $\sigma \in V\left(Y_{1}\right)$, with $\sigma$ of type $T$, we choose $\bar{\sigma}$ in $\Sigma$ to be the vertex of $(1, K)=K \subset \Sigma$ of type $T$.

Let $G(Y)$ be a complex of groups. The fundamental group of the complex of groups $\pi_{1}(G(Y))$ is defined so that if $Y$ is simply connected and $G(Y)$ is simple, $\pi_{1}(G(Y))$ is isomorphic to the direct limit of the family of groups $G_{\sigma}$ and monomorphisms $\psi_{a}$. For example, since $Y_{1}=K$ is contractible and $G\left(Y_{1}\right)$ is a simple complex of groups, the fundamental group of $G\left(Y_{1}\right)$ is $W$.

If $G(Y)$ is a developable complex of groups, then it has a universal cover $\widetilde{G(Y)}$. This is a connected, simply-connected scwol, equipped with an action of $\pi_{1}(G(Y))$, so that the complex of groups induced by the action of the fundamental group on the universal cover is isomorphic to $G(Y)$. For example, the universal cover of $G\left(Y_{1}\right)$ is $\Sigma$.

Let $G(Y)$ be a developable complex of groups over $Y$, with universal cover $X$ and fundamental group $\Gamma$. We say that $G(Y)$ is faithful if the action of $\Gamma$ on $X$ is faithful, in which case we may identify $\Gamma$ with its image in $\operatorname{Aut}(X)$. If $X$ is locally finite, then with the compact-open topology on $\operatorname{Aut}(X)$, by the discussion in Section 2.1 above the subgroup $\Gamma \leq \operatorname{Aut}(X)$ is discrete if and only if all local groups of $G(Y)$ are finite. 
Moreover, if $\operatorname{Aut}(X)$ acts cocompactly on $X$, a discrete $\Gamma \leq \operatorname{Aut}(X)$ is a uniform lattice in $\operatorname{Aut}(X)$ if and only if $Y \cong \Gamma \backslash X$ is finite, and a discrete $\Gamma \leq \operatorname{Aut}(X)$ is a nonuniform lattice if and only if $Y \cong \Gamma \backslash X$ is infinite and the series $\operatorname{Vol}(\Gamma \rrbracket V)$ converges, for some $V \subset X$ on which $G=\operatorname{Aut}(X)$ acts according to the hypotheses of Theorem 2.1 above.

\section{Group actions on complexes of groups}

In this section we introduce a theory of group actions on complexes of groups. The main result is Theorem 3.1 below, which makes precise and expands upon Theorem 1.3 of the introduction. The terms appearing in Theorem 3.1 which have not already been discussed in Section 2.3 above will be defined in Section 3.1 below, where we also introduce some notation. In Section 3.2 below we construct the complex of groups induced by a group action on a complex of groups, and in Section 3.3 we construct the induced covering. Using these results, in Section 3.4 we consider the structure of the fundamental group of the induced complex of groups.

We will require only actions on simple complexes of groups $G(Y)$ by simple morphisms; this case is already substantially technical. If in addition the action on $Y$ has a strict fundamental domain, it is possible to make choices so that the induced complex of groups is also simple, and many of the proofs in this section become much easier. However, in our applications, the group action will not necessarily have a strict fundamental domain.

Theorem 3.1. Let $G(Y)$ be a simple complex of groups over a connected scwol $Y$, and suppose that the action of a group $H$ on $Y$ extends to an action by simple morphisms on $G(Y)$. Then the $H$-action induces a complex of groups $H(Z)$ over $Z=H \backslash Y$, with $H(Z)$ well defined up to isomorphism of complexes of groups, such that there is a covering of complexes of groups

$$
G(Y) \rightarrow H(Z)
$$

Moreover there is a natural short exact sequence

$$
1 \rightarrow \pi_{1}(G(Y)) \rightarrow \pi_{1}(H(Z)) \rightarrow H \rightarrow 1,
$$

and if $H$ fixes a vertex of $Y$, then

$$
\pi_{1}(H(Z)) \cong \pi_{1}(G(Y)) \rtimes H .
$$

Finally, if $G(Y)$ is faithful and the $H$-action on $G(Y)$ is faithful, then $H(Z)$ is faithful.

We will use the following general result on functoriality of coverings (which is implicit in [8], and stated and proved explicitly in [22]). 
Theorem 3.2. Let $G(Y)$ and $H(Z)$ be complexes of groups over scwols $Y$ and $Z$. Suppose there is a covering of complexes of groups $\Phi: G(Y) \rightarrow H(Z)$. Then $G(Y)$ is developable if and only if $H(Z)$ is developable. Moreover, $\Phi$ induces a monomorphism of fundamental groups

$$
\pi_{1}(G(Y)) \hookrightarrow \pi_{1}(H(Z))
$$

and an equivariant isomorphism of universal covers

$$
\widetilde{G(Y)} \stackrel{\cong}{H(Z)} \text {. }
$$

3.1. Definitions and notation. We gather here definitions and notation needed for the statement and proof of Theorem 3.1 above. Throughout this section, $Y$ and $Z$ are scwols, $G(Y)=\left(G_{\sigma}, \psi_{a}\right)$ is a simple complex of groups over $Y$, and $H(Z)=$ $\left(H_{\tau}, \theta_{a}, h_{a, b}\right)$ is a complex of groups over $Z$.

Definition 3.3. Let $f: Y \rightarrow Z$ be a morphism of scwols (see Definition 2.3 above). A morphism $\Phi: G(Y) \rightarrow H(Z)$ over $f$ consists of

(1) a homomorphism $\phi_{\sigma}: G_{\sigma} \rightarrow H_{f(\sigma)}$ for each $\sigma \in V(Y)$, called the local map at $\sigma ;$ and

(2) an element $\phi(a) \in H_{t(f(a))}$ for each $a \in E(Y)$ such that the diagram

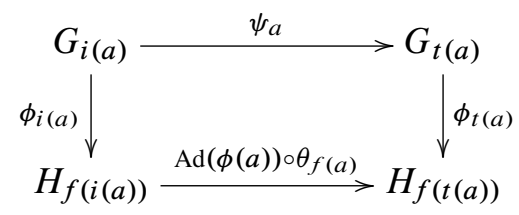

commutes and for all pairs of composable edges $(a, b)$ in $E(Y)$,

$$
\phi(a b)=\phi(a) \psi_{a}(\phi(b)) h_{f(a), f(b)} .
$$

A morphism is simple if each element $\phi(a)$ is trivial. If $f$ is an isomorphism of scwols, and each $\phi_{\sigma}$ an isomorphism of the local groups, then $\Phi$ is an isomorphism of complexes of groups.

We introduce the following, expected, definitions. An automorphism of $G(Y)$ is an isomorphism $\Phi: G(Y) \rightarrow G(Y)$. It is not hard to verify that the set of automorphisms of $G(Y)$ forms a group under composition, which we denote $\operatorname{Aut}(G(Y))$ (see Section III.C.2.4 of [8] for the definition of composition of morphisms). We then say that a group $H$ acts on $G(Y)$ if there is a homomorphism

$$
H \rightarrow \operatorname{Aut}(G(Y)) \text {. }
$$

Our notation is as follows. Suppose $H$ acts on $G(Y)$. Then in particular, $H$ acts on the scwol $Y$ in the sense of Definition 2.4 above. We write the action of $H$ on $Y$ as 
$\sigma \mapsto h \cdot \sigma$ and $a \mapsto h \cdot a$, for $h \in H, \sigma \in V(Y)$ and $a \in E(Y)$. The element $h \in H$ induces the automorphism $\Phi^{h}$ of $G(Y)$. The data for $\Phi^{h}$ is a family $\left(\phi_{\sigma}^{h}\right)_{\sigma \in V(Y)}$ of group isomorphisms $\phi_{\sigma}^{h}: G_{\sigma} \rightarrow G_{h \cdot \sigma}$, and a family of elements $\left(\phi^{h}(a)\right)_{a \in E(Y)}$ with $\phi^{h}(a) \in G_{t(h \cdot a)}$, satisfying the definition of morphism above (Definition 3.3).

We say that the $H$-action is by simple morphisms if each $\Phi^{h}$ is simple, that is, if each $\phi^{h}(a) \in G_{t(h \cdot a)}$ is the trivial element. Explicitly, for each $a \in E(Y)$ and each $h \in H$, the diagram

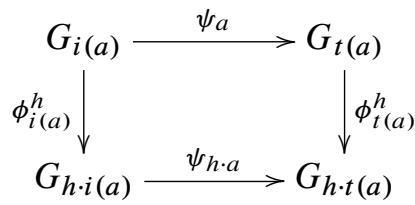

commutes.

We note also that the composition of simple morphisms $\Phi^{h^{\prime}} \circ \Phi^{h}$ is the simple morphism $\Phi^{h^{\prime} h}$ with local maps

$$
\phi_{\sigma}^{h^{\prime} h}=\phi_{h \cdot \sigma}^{h^{\prime}} \circ \phi_{\sigma}^{h} .
$$

Finally we recall the definition of a covering of complexes of groups.

Definition 3.4. A morphism $\Phi: G(Y) \rightarrow H(Z)$ over a nondegenerate morphism of scwols $f: Y \rightarrow Z$ (see Definition 2.3 above) is a covering of complexes of groups if further:

(1) each $\phi_{\sigma}$ is injective; and

(2) for each $\sigma \in V(Y)$ and $b \in E(Z)$ such that $t(b)=f(\sigma)$, the map on cosets

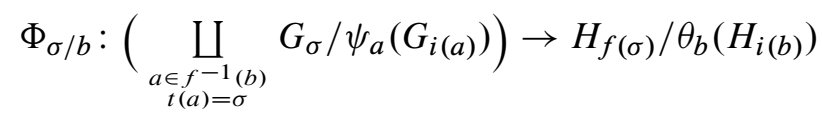

induced by $g \mapsto \phi_{\sigma}(g) \phi(a)$ is a bijection.

3.2. The induced complex of groups and its properties. Suppose that a group $H$ acts by simple morphisms on a simple complex of groups $G(Y)=\left(G_{\sigma}, \psi_{a}\right)$. In this section we construct the complex of groups $H(Z)$ induced by this action, prove that $H(Z)$ is well defined up to isomorphism of complexes of groups and discuss faithfulness.

Let $Z$ be the quotient scwol $Z=H \backslash Y$ and let $p: Y \rightarrow Z$ be the natural projection. For each vertex $\tau \in V(Z)$ choose a representative $\bar{\tau} \in V(Y)$ such that $p(\bar{\tau})=\tau$. Let $\operatorname{Stab}_{H}(\bar{\tau})$ be the subgroup of $H$ fixing $\bar{\tau}$ and let $G_{\bar{\tau}}$ be the local group of $G(Y)$ at $\bar{\tau}$. Since the $H$-action is by simple morphisms, by equation (1) above there is a group homomorphism $\zeta: \operatorname{Stab}_{H}(\bar{\tau}) \rightarrow \operatorname{Aut}\left(G_{\bar{\tau}}\right)$ given by $\zeta(h)=\phi_{\bar{\tau}}^{h}$. For each $\tau \in V(Z)$ we then define the local group $H_{\tau}$ to be the corresponding semidirect product of $G_{\bar{\tau}}$ by $\operatorname{Stab}_{H}(\bar{\tau})$, that is,

$$
H_{\tau}:=G_{\bar{\tau}} \rtimes_{\zeta} \operatorname{Stab}_{H}(\bar{\tau})=G_{\bar{\tau}} \rtimes \operatorname{Stab}_{H}(\bar{\tau}) .
$$


For each edge $a \in E(Z)$ with $i(a)=\tau$ there is, since $H$ acts on $Y$ in the sense of Definition 2.4 above, a unique edge $\bar{a} \in E(Y)$ such that $p(\bar{a})=a$ and $i(\bar{a})=\overline{i(a)}=\bar{\tau}$. For each $a \in E(Z)$ choose an element $h_{a} \in H$ such that $h_{a} \cdot t(\bar{a})=\overline{t(a)}$.

Lemma 3.5. Let $g \in G_{i(\bar{a})}=G_{\overline{i(a)}}$ and $h \in \operatorname{Stab}_{H}(\overline{i(a)})$. Then the map

$$
\theta_{a}:(g, h) \mapsto\left(\phi_{t(\bar{a})}^{h_{a}} \circ \psi_{\bar{a}}(g), h_{a} h h_{a}^{-1}\right)
$$

is a monomorphism $H_{i(a)} \rightarrow H_{t(a)}$.

Proof. We will show that $\theta_{a}$ is a group homomorphism. Since $\phi_{t(\bar{a})}^{h_{a}}, \psi_{\bar{a}}$ and the conjugation $h \mapsto h_{a} h h_{a}^{-1}$ are all injective, the conclusion will then follow.

Let $g, g^{\prime} \in G_{\overline{i(a)}}$ and $h, h^{\prime} \in \operatorname{Stab}_{H}(\overline{i(a)})$. Note that since $h$ and $h^{\prime}$ fix $\overline{i(a)}=$ $i(\bar{a})$, they fix the edge $\bar{a}$ and hence fix the vertex $t(\bar{a})$ as well. We have

$$
\theta_{a}\left((g, h)\left(g, h^{\prime}\right)\right)=\theta_{a}\left(g \phi_{\overline{i(a)}}^{h}\left(g^{\prime}\right), h h^{\prime}\right)=\left(\phi_{t(\bar{a})}^{h_{a}} \circ \psi_{\bar{a}}\left(g \phi_{\bar{i}(a)}^{h}\left(g^{\prime}\right)\right), h_{a} h h^{\prime} h_{a}^{-1}\right)
$$

while

$$
\begin{aligned}
\theta_{a}(g, h) \theta_{a}\left(g^{\prime}, h^{\prime}\right) & =\left(\phi_{t(\bar{a})}^{h_{a}} \circ \psi_{\bar{a}}(g), h_{a} h h_{a}^{-1}\right)\left(\phi_{t(\bar{a})}^{h_{a}} \circ \psi_{\bar{a}}\left(g^{\prime}\right), h_{a} h^{\prime} h_{a}^{-1}\right) \\
& =\left(\phi_{t(\bar{a})}^{h_{a}} \circ \psi_{\bar{a}}(g) \phi_{\bar{t}(a)}^{h_{a} h h_{a}^{-1}} \circ \phi_{t(\bar{a})}^{h_{a}} \circ \psi_{\bar{a}}\left(g^{\prime}\right), h_{a} h h^{\prime} h_{a}^{-1}\right) .
\end{aligned}
$$

After applying equation (1) above to the map $\phi^{h_{a}} h h_{a}^{-1}$, and some cancellations, it remains to show that

$$
\psi_{\bar{a}} \circ \phi_{\overline{i(a)}}^{h}\left(g^{\prime}\right)=\phi_{t(\bar{a})}^{h} \circ \psi_{\bar{a}}\left(g^{\prime}\right) .
$$

This follows from the fact that $\Phi^{h}$ is a simple morphism with $h \cdot \bar{a}=\bar{a}$.

To complete the construction of $H(Z)$, for each composable pair of edges $(a, b)$ in $E(Z)$, define

$$
h_{a, b}=h_{a} h_{b} h_{a b}^{-1} \text {. }
$$

One checks that $\left.h_{a, b} \in \operatorname{Stab}_{H}(\overline{t(a})\right)$ hence $\left(1, h_{a, b}\right) \in H_{t(a)}$. By abuse of notation we write $h_{a, b}$ for $\left(1, h_{a, b}\right)$.

Proposition 3.6. The datum $H(Z)=\left(H_{\sigma}, \theta_{a}, h_{a, b}\right)$ is a complex of groups.

Proof. Given Lemma 3.5 above, it remains to show that for each pair of composable edges $(a, b)$ in $E(Z)$,

$$
\operatorname{Ad}\left(h_{a, b}\right) \circ \theta_{a b}=\theta_{a} \circ \theta_{b},
$$

and that the cocycle condition holds. Let $(g, h) \in H_{i(b)}=G_{\overline{i(b)}} \rtimes \operatorname{Stab}_{H}(\overline{i(b)})$. We compute

$$
\operatorname{Ad}\left(h_{a, b}\right) \circ \theta_{a b}(g, h)=\left(\phi_{\frac{t(a b)}{h_{a, b}}} \circ \phi_{t(\overline{a b})}^{h_{a b}} \circ \psi_{\overline{a b}}(g), h_{a, b} h_{a b} h h_{a b}^{-1} h_{a, b}^{-1}\right)
$$


while

$$
\theta_{a} \circ \theta_{b}(g, h)=\left(\phi_{t(\bar{a})}^{h_{a}} \circ \psi_{\bar{a}} \circ \phi_{t(\bar{b})}^{h_{b}} \circ \psi_{\bar{b}}(g), h_{a} h_{b} h h_{b}^{-1} h_{a}^{-1}\right) .
$$

By definition of $h_{a, b}$ it remains to show equality in the first component.

By equation (1) and the definition of $h_{a, b}$,

$$
\phi_{t(a b)}^{h_{a, b}}=\phi_{t(\bar{a})}^{h_{a}} \circ \phi_{t(\overline{a b})}^{h_{b}} \circ \phi_{t(a b)}^{h_{a b}^{-1}} .
$$

Hence it suffices to prove

$$
\phi_{t(\overline{a b})}^{h_{b}} \circ \psi_{\overline{a b}}=\psi_{\bar{a}} \circ \phi_{t(\bar{b})}^{h_{b}} \circ \psi_{\bar{b}}
$$

Since $G(Y)$ is a simple complex of groups, and $\overline{a b}$ is the composition of the edges $h_{b}^{-1} \cdot \bar{a}$ and $\bar{b}$, we have

$$
\psi_{\overline{a b}}=\psi_{h_{b}^{-1} \bar{a}} \circ \psi_{\bar{b}}
$$

Applying this, and the fact that $\phi_{t(\bar{b})}^{h_{b}}$ is a simple morphism on the edge $h_{b}^{-1} \bar{a}$, we have

$$
\phi_{t(\overline{a b})}^{h_{b}} \circ \psi_{\overline{a b}}=\phi_{t(\overline{a b})}^{h_{b}} \circ \psi_{h_{b}^{-1} \bar{a}} \circ \psi_{\bar{b}}=\psi_{\bar{a}} \circ \phi_{t(\bar{b})}^{h_{b}} \circ \psi_{\bar{b}}
$$

Hence equation (2) holds.

The cococycle condition follows from the definition of $h_{a, b}$. We conclude that $H(Z)$ is a complex of groups.

We now have a complex of groups $H(Z)$ induced by the action of $H$ on $G(Y)$. This construction depended on choices of lifts $\bar{\tau}$ and of elements $h_{a} \in H$. We next show (in a generalisation of Section III.C.2.9(2) of [8]) that:

Lemma 3.7. The complex of groups $H(Z)$ is well defined up to isomorphism of complexes of groups.

Proof. Suppose we made a different choice of lifts $\bar{\tau}^{\prime}$ and elements $h_{a}^{\prime}$, resulting in a complex of groups $H^{\prime}(Z)=\left(H_{\tau}^{\prime}, \theta_{a}^{\prime}, h_{a, b}^{\prime}\right)$. An isomorphism $\Lambda=\left(\lambda_{\sigma}, \lambda(a)\right)$ from $H(Z)$ to $H^{\prime}(Z)$ over the identity map $Z \rightarrow Z$ is constructed as follows. For each $\tau \in V(Z)$, choose an element $k_{\tau} \in H$ such that $k_{\tau} \cdot \bar{\tau}=\bar{\tau}^{\prime}$, and define a group isomorphism $\lambda_{\tau}: H_{\tau} \rightarrow H_{\tau}^{\prime}$ by

$$
\lambda_{\tau}(g, h)=\left(\phi_{\bar{\tau}}^{k_{\tau}}(g), k_{\tau} h k_{\tau}^{-1}\right) .
$$

For each $a \in E(Z)$, define $\lambda(a)=\left(1, k_{t(a)} h_{a} k_{i(a)}^{-1} h_{a}^{\prime-1}\right)$. Note that by III.C.2.9(2) of [8], $\lambda(a) \in H_{t(a)}^{\prime}$.

The verification that $\Lambda=\left(\lambda_{\sigma}, \lambda(a)\right)$ is an isomorphism of complexes of groups is straightforward. 
We remind the reader that faithfulness of a complex of groups is defined in the final paragraph of Section 2.3 above.

Lemma 3.8. If $G(Y)$ is faithful and the $H$-action on $Y$ is faithful then $H(Z)$ is faithful.

Proof. This follows from the construction of $H(Z)$, and the characterisation of faithful complexes of groups in Proposition 38 of [22].

3.3. The induced covering. Suppose $H$ acts by simple morphisms on a simple complex of groups $G(Y)$, inducing a complex of groups $H(Z)$ as in Section 3.2 above. In this section we construct a covering of complexes of groups $\Lambda: G(Y) \rightarrow H(Z)$ over the quotient map $p: Y \rightarrow Z$.

For $\sigma \in V(Y)$, the local maps $\lambda_{\sigma}: G_{\sigma} \rightarrow H_{p(\sigma)}$ are defined as follows. Recall that for each vertex $\tau \in V(Z)$ we chose a lift $\bar{\tau} \in V(Y)$. Now for each $\sigma \in V(Y)$, we choose $k_{\sigma} \in H$ such that $k_{\sigma} \cdot \sigma=\overline{p(\sigma)}$. Hence $\phi_{\sigma}^{k_{\sigma}}$ is an isomorphism $G_{\sigma} \rightarrow G_{\overline{p(\sigma)}}$. The local map $\lambda_{\sigma}: G_{\sigma} \rightarrow H_{p(\sigma)}$ is then defined by

$$
\lambda_{\sigma}: g \mapsto\left(\phi_{\sigma}^{k_{\sigma}}(g), 1\right) .
$$

Note that each $\lambda_{\sigma}$ is injective.

For each edge $a \in E(Y)$, define

$$
\lambda(a)=\left(1, k_{t(a)} k_{i(a)}^{-1} h_{b}^{-1}\right)
$$

where $p(a)=b \in E(Z)$. Note that since $H$ acts on $Y$ in the sense of Definition 2.4 above, we have $k_{i(a)} \cdot a=\bar{b}$ hence $k_{t(a)} k_{i(a)}^{-1} h_{b}^{-1}$ fixes $\overline{t(b)}$. Thus $\lambda(a) \in H_{t(b)}$ as required.

Proposition 3.9. The map $\Lambda=\left(\lambda_{\sigma}, \lambda(a)\right)$ is a covering of complexes of groups.

Proof. It may be checked that $\Lambda$ is a morphism of complexes of groups. As noted, each of the local maps $\lambda_{\sigma}$ is injective. It remains to show that for each $\sigma \in V(Y)$ and $b \in E(Z)$ such that $t(b)=p(\sigma)=\tau$, the map on cosets

$$
\Lambda_{\sigma / b}:\left(\coprod_{\substack{a \in p^{-1}(b) \\ t(a)=\sigma}} G_{\sigma} / \psi_{a}\left(G_{i(a)}\right)\right) \rightarrow H_{\tau} / \theta_{b}\left(H_{i(b)}\right)
$$

induced by $g \mapsto \lambda_{\sigma}(g) \lambda(a)=\left(\phi_{\sigma}^{k_{\sigma}}(g), k_{\sigma} k_{i(a)}^{-1} h_{b}^{-1}\right)$ is a bijection.

We first show that $\Lambda_{\sigma / b}$ is injective. Suppose $a$ and $a^{\prime}$ are in $p^{-1}(b)$ with $t(a)=$ $t\left(a^{\prime}\right)=\sigma$, and suppose $g, g^{\prime} \in G_{\sigma}$ with $g$ representing a coset of $\psi_{a}\left(G_{i(a)}\right)$ in $G_{\sigma}$ and $g^{\prime}$ a coset of $\psi_{a^{\prime}}\left(G_{i\left(a^{\prime}\right)}\right)$ in $G_{\sigma}$. Assume that $\lambda_{\sigma}(g) \lambda(a)$ and $\lambda_{\sigma}\left(g^{\prime}\right) \lambda\left(a^{\prime}\right)$ belong to the same coset of $\theta_{b}\left(H_{i(b)}\right)$ in $H_{\tau}$. 
Looking at the second component of the semidirect product $H_{\tau}$, it follows from the definition of $\theta_{b}$ (Lemma 3.5 above) that for some $h \in \operatorname{Stab}_{H}(\overline{i(b)})$,

$$
k_{\sigma} k_{i(a)}^{-1} h_{b}^{-1}=\left(k_{\sigma} k_{i\left(a^{\prime}\right)}^{-1} h_{b}^{-1}\right)\left(h_{b} h h_{b}^{-1}\right)=k_{\sigma} k_{i\left(a^{\prime}\right)}^{-1} h h_{b}^{-1} .
$$

Thus $k_{i\left(a^{\prime}\right)} k_{i(a)}^{-1}=h$ fixes $\overline{i(b)}$. Hence $k_{i(a)}^{-1} k_{i\left(a^{\prime}\right)}$ fixes $k_{i(a)}^{-1} \overline{i(b)}=i(a)$, and so $k_{i(a)}^{-1} k_{i\left(a^{\prime}\right)}$ fixes $a$. Thus $k_{i\left(a^{\prime}\right)} \cdot a=k_{i(a)} \cdot a=\bar{b}=k_{i\left(a^{\prime}\right)} \cdot a^{\prime}$, hence $a=a^{\prime}$.

Looking now at the first component of $\lambda_{\sigma}(g) \lambda(a)$ and $\lambda_{\sigma}\left(g^{\prime}\right) \lambda\left(a^{\prime}\right)=\lambda_{\sigma}\left(g^{\prime}\right) \lambda(a)$ in the semidirect product $H_{\tau}$, by definition of $\theta_{b}$, for some $x \in G_{\overline{i(b)}}$ we have

$$
\phi_{\sigma}^{k_{\sigma}}(g)=\phi_{\sigma}^{k_{\sigma}}\left(g^{\prime}\right) \phi_{\overline{t(b)}}^{k_{\sigma} k_{i(a)}^{-1} h_{b}^{-1}} \circ \phi_{t(\bar{b})}^{h_{b}} \circ \psi_{\bar{b}}(x)=\phi_{\sigma}^{k_{\sigma}}\left(g^{\prime}\right) \phi_{\sigma}^{k_{\sigma}} \circ \phi_{t(\bar{b})}^{k_{i(a)}^{-1}} \circ \psi_{\bar{b}}(x) .
$$

Since $\phi_{\sigma}^{k_{\sigma}}$ is an isomorphism, and $k_{i(a)}^{-1} \cdot \bar{b}=a$, this implies that

$$
\left(g^{\prime}\right)^{-1} g=\phi_{t(\bar{b})}^{k_{i(a)}^{-1}} \circ \psi_{\bar{b}}(x)=\psi_{a} \circ \phi_{i(b)}^{k_{i(a)}^{-1}}(x) \in \psi_{a}\left(G_{i(a)}\right)
$$

as required. Thus the map $\Lambda_{\sigma / b}$ is injective.

To show that $\Lambda_{\sigma / b}$ is surjective, let $g \in G_{\bar{\tau}}$ and $h \in \operatorname{Stab}_{H}(\bar{\tau})$, so that $(g, h) \in H_{\tau}$. Let $a$ be the unique edge of $Y$ with $t(a)=\sigma$ and such that $k_{\sigma} \cdot a=h h_{b} \bar{b}$. Let $g^{\prime}$ be the unique element of $G_{\sigma}$ such that $\phi_{\sigma}^{k_{\sigma}}\left(g^{\prime}\right)=g \in G_{\bar{\tau}}$. We claim that $\lambda_{\sigma}\left(g^{\prime}\right) \lambda(a)$ lies in the same coset as $(g, h)$. Now

$$
\lambda_{\sigma}\left(g^{\prime}\right) \lambda(a)=\left(\phi_{\sigma}^{k_{\sigma}}\left(g^{\prime}\right), k_{\sigma} k_{i(a)}^{-1} h_{b}^{-1}\right)=\left(g, k_{\sigma} k_{i(a)}^{-1} h_{b}^{-1}\right),
$$

so it suffices to show that $k_{\sigma} k_{i(a)}^{-1} h_{b}^{-1} \in h h_{b} \operatorname{Stab}_{H}(\overline{i(b)}) h_{b}^{-1}$. Equivalently, we wish to show that $h_{b}^{-1} h^{-1} k_{\sigma} k_{i(a)}^{-1}$ fixes $\overline{i(b)}$. We have $k_{i(a)} \cdot i(a)=\overline{i(b)}$ by definition, and the result follows by our choice of $a$. Thus $\Lambda_{\sigma / b}$ is surjective.

Hence $\Lambda$ is a covering of complexes of groups.

3.4. The fundamental group. Suppose $H$ acts by simple morphisms on a simple complex of groups $G(Y)$, inducing a complex of groups $H(Z)$ as in Section 3.2 above. In this section we establish the short exact sequence of Theorem 3.1 above, and provide sufficient conditions for the fundamental group of $H(Z)$ to be the semidirect product of the fundamental group of $G(Y)$ by $H$.

Fix $\sigma_{0}$ a vertex of $Y$ and let $p: Y \rightarrow Z$ be the natural projection. We refer the reader to Section III. .3 of [8] for the definition of the fundamental group of $G(Y)$ at $\sigma_{0}$, denoted by $\pi_{1}\left(G(Y), \sigma_{0}\right)$. We will use notation and results from that section in the following proof. Let $\pi_{1}\left(H(Z), p\left(\sigma_{0}\right)\right)$ be the fundamental group of $H(Z)$ at $p\left(\sigma_{0}\right)$.

Proposition 3.10. There is a natural short exact sequence

$$
1 \rightarrow \pi_{1}\left(G(Y), \sigma_{0}\right) \rightarrow \pi_{1}\left(H(Z), p\left(\sigma_{0}\right)\right) \rightarrow H \rightarrow 1 .
$$


Proof. To obtain a monomorphism $\pi_{1}\left(G(Y), \sigma_{0}\right) \rightarrow \pi_{1}\left(H(Z), p\left(\sigma_{0}\right)\right)$, we use the morphism of complexes of groups $\Lambda: G(Y) \rightarrow H(Z)$ defined in Section 3.3 above. By Proposition III.C.3.6 of [8], $\Lambda$ induces a natural homomorphism

$$
\pi_{1}\left(\Lambda, \sigma_{0}\right): \pi_{1}\left(G(Y), \sigma_{0}\right) \rightarrow \pi_{1}\left(H(Z), p\left(\sigma_{0}\right)\right)
$$

Since $\Lambda$ is a covering (Proposition 3.9 above), Theorem 3.2 above implies that this map $\pi_{1}\left(\Lambda, \sigma_{0}\right)$ is in fact injective.

We next define a surjection $\pi_{1}\left(H(Z), p\left(\sigma_{0}\right)\right) \rightarrow H$. The group $H$ may be regarded as a complex of groups over a single vertex. There is then a canonical morphism of complexes of groups $\Phi: H(Z) \rightarrow H$, defined as follows. Recall that for each $\tau \in V(Z)$, the local group $H_{\tau}$ is given by $H_{\tau}=G_{\bar{\tau}} \rtimes \operatorname{Stab}_{H}(\bar{\tau})$. The local map $\phi_{\tau}: H_{\tau} \rightarrow H$ in the morphism $\Phi$ is defined to be projection to the second factor $\operatorname{Stab}_{H}(\bar{\tau}) \leq H$. For each edge $b$ of $Z$, we define $\phi(b)=h_{b}$. It may then be checked that $\Phi$ is a morphism.

By Proposition III.C.3.6 of [8], the morphism $\Phi$ induces a homomorphism of fundamental groups

$$
\pi_{1}\left(\Phi, p\left(\sigma_{0}\right)\right): \pi_{1}\left(H(Z), p\left(\sigma_{0}\right)\right) \rightarrow H
$$

By III.C.3.14 and Corollary III.C.3.15 of [8], if $G(Y)$ were a complex of trivial groups, this map would be surjective. Since the image of $\pi_{1}\left(\Phi, p\left(\sigma_{0}\right)\right)$ does not in fact depend on the local groups of $G(Y)$, we have that in all cases, $\pi_{1}\left(\Phi, p\left(\sigma_{0}\right)\right)$ is surjective, as required.

It follows from definitions that the image of the monomorphism $\pi_{1}\left(\Lambda, \sigma_{0}\right)$ is the kernel of the surjection $\pi_{1}\left(\Phi, p\left(\sigma_{0}\right)\right)$. Hence the sequence above is exact.

Corollary 3.11. If $H$ fixes a vertex of $Y$,

$$
\pi_{1}\left(H(Z), p\left(\sigma_{0}\right)\right) \cong \pi_{1}\left(G(Y), \sigma_{0}\right) \rtimes H
$$

Proof. Suppose that $H$ fixes the vertex $\sigma$ of $Y$. For the surjective homomorphism $\pi_{1}\left(\Phi, p\left(\sigma_{0}\right)\right): \pi_{1}\left(H(Z), p\left(\sigma_{0}\right)\right) \rightarrow H$ given in the proof of Proposition 3.10 above, we construct a section $\iota: H \rightarrow \pi_{1}\left(H(Z), p\left(\sigma_{0}\right)\right)$.

The vertex $\sigma$ is the unique lift $\bar{\tau}$ of a vertex $p(\sigma)=\tau \in Z$. Hence

$$
H_{\tau}=G_{\bar{\tau}} \rtimes \operatorname{Stab}_{H}(\bar{\tau})=G_{\sigma} \rtimes H .
$$

By definition of the surjection $\pi_{1}\left(\Phi, p\left(\sigma_{0}\right)\right): \pi_{1}\left(H(Z), p\left(\sigma_{0}\right)\right) \rightarrow H$, a section $\iota: H \rightarrow \pi_{1}\left(H(Z), p\left(\sigma_{0}\right)\right)$ is then given by the inclusion $H \rightarrow H_{\tau}$.

This completes the proof of Theorem 3.1. 


\section{Proof of the Main Theorem}

We now prove the Main Theorem and Corollary 1.2, stated in the introduction. Throughout this section, we adopt the notation of the Main Theorem, and assume that the vertices $s_{1}$ and $s_{2}$ of the nerve $L$, and the elements $\alpha_{1}$ and $\alpha_{2}$ of the group $A$ of label-preserving automorphisms of $L$, satisfy conditions (1)-(4) of its statement. In Section 4.1 we introduce notation, and construct a family of finite polyhedral complexes $Y_{n}$, for $n \geq 1$, and an infinite polyhedral complex $Y_{\infty}$. We then in Section 4.2 construct complexes of groups $G\left(Y_{n}\right)$ and $G\left(Y_{\infty}\right)$ over these spaces, and show that there are coverings of complexes of groups $G\left(Y_{n}\right) \rightarrow G\left(Y_{1}\right)$ and $G\left(Y_{\infty}\right) \rightarrow G\left(Y_{1}\right)$. In Section 4.3 we define the action of a finite group $H_{n}$ on $Y_{n}$, and of an infinite group $H_{\infty}$ on $Y_{\infty}$, and then in Section 4.4 we show that these actions extend to actions on the complexes of groups $G\left(Y_{n}\right)$ and $G\left(Y_{\infty}\right)$. In Section 4.5 we combine these results with Theorem 3.1 above to complete the proof of the Main Theorem. Corollary 1.2 is proved in Section 4.6.

4.1. The spaces $Y_{n}$ and $Y_{\infty}$. In this section we construct a family of finite polyhedral complexes $Y_{n}$ and an infinite polyhedral complex $Y_{\infty}$.

We first set up some notation. For $i=1,2$, let $q_{i} \geq 2$ be the order of $\alpha_{i}$. It will be convenient to put, for all $k \geq 0, s_{2 k+1}=s_{1}$ and $s_{2 k+2}=s_{2}$, and similarly $\alpha_{2 k+1}=\alpha_{1}, \alpha_{2 k+2}=\alpha_{2}, q_{2 k+1}=q_{1}$ and $q_{2 k+2}=q_{2}$. Conditions (1)-(4) of the Main Theorem then become:

(1) for all $n \geq 1, \alpha_{n}$ fixes the star of $s_{n+1}$ in $L$;

(2) for all $n \geq 1$, the subgroup $\left\langle\alpha_{n}\right\rangle$ of $A$ acts freely on the $\left\langle\alpha_{n}\right\rangle$-orbit of $s_{n}$, in particular $\alpha_{n}\left(s_{n}\right) \neq s_{n}$

(3) for all $n \geq 1$, and all $t_{n} \neq s_{n}$ such that $t_{n}$ is in the $\left\langle\alpha_{n}\right\rangle$-orbit of $s_{n}, m_{s_{n} t_{n}}=\infty$; and

(4) for all $n \geq 1$, all spherical special subgroups of $W$ which contain $s_{n}$ are halvable along $s_{n}$.

We now use the sequences $\left\{s_{n}\right\}$ and $\left\{\alpha_{n}\right\}$ to define certain elements and subsets of $W$. Let $w_{1}$ be the trivial element of $W$ and for $n \geq 2$ let $w_{n}$ be the product

$$
w_{n}=s_{1} s_{2} \ldots s_{n-1} \in W .
$$

Denote by $W_{n, n}$ the one-element set $\left\{w_{n}\right\}$. For $n \geq 2$, and $1 \leq k<n$, in order to simplify notation, write $\alpha^{j_{n-1}, \ldots, j_{k}}$ for the composition of automorphisms

$$
\alpha^{j_{n-1}, \ldots, j_{k}}=\alpha_{n-1}^{j_{n-1}} \ldots \alpha_{k}^{j_{k}}
$$

where $0 \leq j_{i}<q_{i}$ for $k \leq i<n$. Let $w_{j_{n-1}, \ldots, j_{k}}$ be the element of $W$ :

$$
\begin{aligned}
& w_{j_{n-1}, \ldots, j_{k}} \\
& \quad=w_{n} \alpha^{j_{n-1}}\left(s_{n-1}\right) \alpha^{j_{n-1}, j_{n-2}}\left(s_{n-2}\right) \ldots \alpha^{j_{n-1}, \ldots, j_{k+1}}\left(s_{k+1}\right) \alpha^{j_{n-1}, \ldots, j_{k}}\left(s_{k}\right) .
\end{aligned}
$$


Now for $n \geq 2$ and $1 \leq k<n$, define

$$
W_{k, n}=\left\{w_{j_{n-1}, \ldots, j_{k}} \in W \mid 0 \leq j_{i}<q_{i} \text { for } k \leq i<n\right\} .
$$

Note that if $j_{n-1}=0$ then $w_{j_{n-1}, \ldots, j_{k}} \in W_{k, n-1}$.

Example. Let $(W, S)$ be the Coxeter system in Example 1 of Section 2.2 above, with nerve $L$ shown in Figure 3 above. For $i=1,2$, let $\alpha_{i} \in A$ be the automorphism of $L$ which fixes the star of $s_{3-i}$ in $L$ and interchanges $s_{i}$ and $s_{3}$. Then if $m$ and $m^{\prime}$ are both even, the Main Theorem applies to this example. (If $T=\{s\}$, then $W_{T}$ is halvable along $s$ with half $s\left(W_{T}\right)$ the trivial group. If $T=\{s, t\}$, then $W_{T}$ is the dihedral group of order $2 m_{s t}$, and $W_{T}$ is halvable along $s$ if and only if $m_{s t}$ is even, in which case half ${ }_{s}\left(W_{T}\right)$ is the dihedral group of order $m_{s t}$.) Note that $q_{1}=q_{2}=2$, and so, for instance,

$$
\begin{aligned}
& W_{1,3}=\left\{1, s_{1} \alpha_{1}\left(s_{1}\right), s_{1} s_{2} \alpha_{2}\left(s_{2}\right) \alpha_{2}\left(s_{1}\right), s_{1} s_{2} \alpha_{2}\left(s_{2}\right) \alpha_{2} \alpha_{1}\left(s_{1}\right)\right\}, \\
& W_{2,3}=\left\{s_{1}, s_{1} s_{2} \alpha_{2}\left(s_{2}\right)\right\}, \\
& W_{3,3}=\left\{s_{1} s_{2}\right\} .
\end{aligned}
$$

The following lemma establishes key properties of the sets $W_{k, n}$.

Lemma 4.1. For all $n \geq 1$ :

(1) The sets $W_{1, n}, W_{2, n}, \ldots, W_{n, n}$ are pairwise disjoint.

(2) For all $1 \leq k<n$, if

$$
w_{j_{n-1}, \ldots, j_{k}}=w_{j_{n-1}^{\prime}, \ldots, j_{k}^{\prime}}
$$

(where $0 \leq j_{i}<q_{i}$ for $k \leq i<n$ ), then $j_{k}=j_{k}^{\prime}, j_{k+1}=j_{k+1}^{\prime}, \ldots$, and $j_{n-1}=j_{n-1}^{\prime}$.

Proof. Given $1 \leq k \leq k^{\prime}<n$, with $0 \leq j_{i}<q_{i}$ for $k \leq i<n$ and $0 \leq j_{i}^{\prime}<q_{i}$ for $k^{\prime} \leq i<n$, suppose that

$$
w_{j_{n-1}, \ldots, j_{k}}=w_{j_{n-1}^{\prime}, \ldots, j_{k^{\prime}}^{\prime}}
$$

Then

$$
\begin{aligned}
& \alpha^{j_{n-1}}\left(s_{n-1}\right) \alpha^{j_{n-1}, j_{n-2}}\left(s_{n-2}\right) \ldots \alpha^{j_{n-1}, \ldots, j_{k^{\prime}}, \ldots, j_{k+1}}\left(s_{k+1}\right) \alpha^{j_{n-1}, \ldots, j_{k^{\prime}}, \ldots, j_{k}}\left(s_{k}\right) \\
& =\alpha^{j_{n-1}^{\prime}}\left(s_{n-1}\right) \alpha^{j_{n-1}^{\prime}, j_{n-2}^{\prime}}\left(s_{n-2}\right) \ldots \alpha^{j_{n-1}^{\prime}, \ldots, j_{k^{\prime}+1}^{\prime}}\left(s_{k^{\prime}+1}\right) \alpha^{j_{n-1}^{\prime}, \ldots, j_{k^{\prime}}^{\prime}}\left(s_{k^{\prime}}\right) .
\end{aligned}
$$

By condition (1) above, for each $k \leq i<n$, the automorphism $\alpha_{i}$ fixes $s_{i+1}$, thus

$$
\begin{aligned}
\alpha^{j_{n-1}, \ldots, j_{i+1}}\left(s_{i+1}\right) \alpha^{j_{n-1}, \ldots, j_{i}}\left(s_{i}\right) & =\alpha^{j_{n-1}, \ldots, j_{i+1}, j_{i}}\left(s_{i+1}\right) \alpha^{j_{n-1}, \ldots, j_{i}}\left(s_{i}\right) \\
& =\alpha^{j_{n-1}, \ldots, j_{i}}\left(s_{i+1} s_{i}\right) .
\end{aligned}
$$


Also since $\alpha_{i}$ fixes the star of $s_{i+1}$ but $\alpha_{i}\left(s_{i}\right) \neq s_{i}$, we have $m_{s_{i+1} s_{i}}=\infty$. Since $\alpha^{j_{n-1}, \ldots, j_{i}}$ is a label-preserving automorphism, it follows that the product of the two generators

$$
\alpha^{j_{n-1}, \ldots, j_{i+1}}\left(s_{i+1}\right) \alpha^{j_{n-1}, \ldots, j_{i}}\left(s_{i}\right)
$$

has infinite order, for each $k \leq i<n$. Similarly for each $k^{\prime} \leq i<n$. Thus the only way for equation (3) to hold is if $k=k^{\prime}$, and for each $k \leq i<n, \alpha_{i}^{j_{i}}\left(s_{i}\right)=\alpha_{i}^{j_{i}^{\prime}}\left(s_{i}\right)$. Since $\left\langle\alpha_{i}\right\rangle$ acts freely on the $\left\langle\alpha_{i}\right\rangle$-orbit of $s_{i}$ and we specified $0 \leq j_{i}<q_{i}$, the result follows.

For $n \geq 1$, and $1 \leq k \leq n$, define $Y_{k, n}$ to be the set of chambers

$$
Y_{k, n}:=\left\{w K \mid w \in W_{k, n}\right\} .
$$

Recall that we are writing $w K$ for the pair $(w, K)$. By Lemma 4.1 above, for fixed $n$, the sets $Y_{1, n}, \ldots, Y_{n, n}$ are pairwise disjoint. We now define $Y_{n}$ to be the polyhedral complex obtained by "gluing together" the chambers in $Y_{1, n}, \ldots, Y_{n, n}$, using the same relation $\sim$ as in the Davis complex $\Sigma$ for $(W, S)$. More precisely,

$$
Y_{n}:=\left(\coprod_{k=1}^{n} Y_{k, n}\right) / \sim
$$

where, for $x, x^{\prime} \in K$, we have $(w, x) \sim\left(w^{\prime}, x^{\prime}\right)$ if and only if $x=x^{\prime}$ and $w^{-1} w^{\prime} \in$ $W_{S(x)}$. Note that $Y_{1}=Y_{1,1}=K$. To define $Y_{\infty}$, for each $k \geq 1$, noting that $W_{k, n}$ is only defined for $1 \leq k \leq n$, put

$$
W_{k, \infty}:=\bigcup_{n=k}^{\infty} W_{k, n}
$$

Then $Y_{k, \infty}$ is the set of chambers

$$
Y_{k, \infty}:=\left\{w K \mid w \in W_{k, \infty}\right\} .
$$

Similarly to the finite case, the sets $Y_{1, \infty}, Y_{2, \infty}, \ldots$ are pairwise disjoint, and we define

$$
Y_{\infty}=\left(\coprod_{k=1}^{\infty} Y_{k, \infty}\right) / \sim
$$

for the same relation $\sim$. Note that there are natural strict inclusions as subcomplexes

$$
Y_{1} \subset Y_{2} \subset \cdots \subset Y_{n} \subset \cdots Y_{\infty} .
$$

(In fact, $Y_{n}$ and $Y_{\infty}$ are subcomplexes of the Davis complex $\Sigma$, but we will not adopt this point of view.) We define a mirror of $Y_{n}$ or $Y_{\infty}$ to be an interior mirror if it is contained in more than one chamber. 


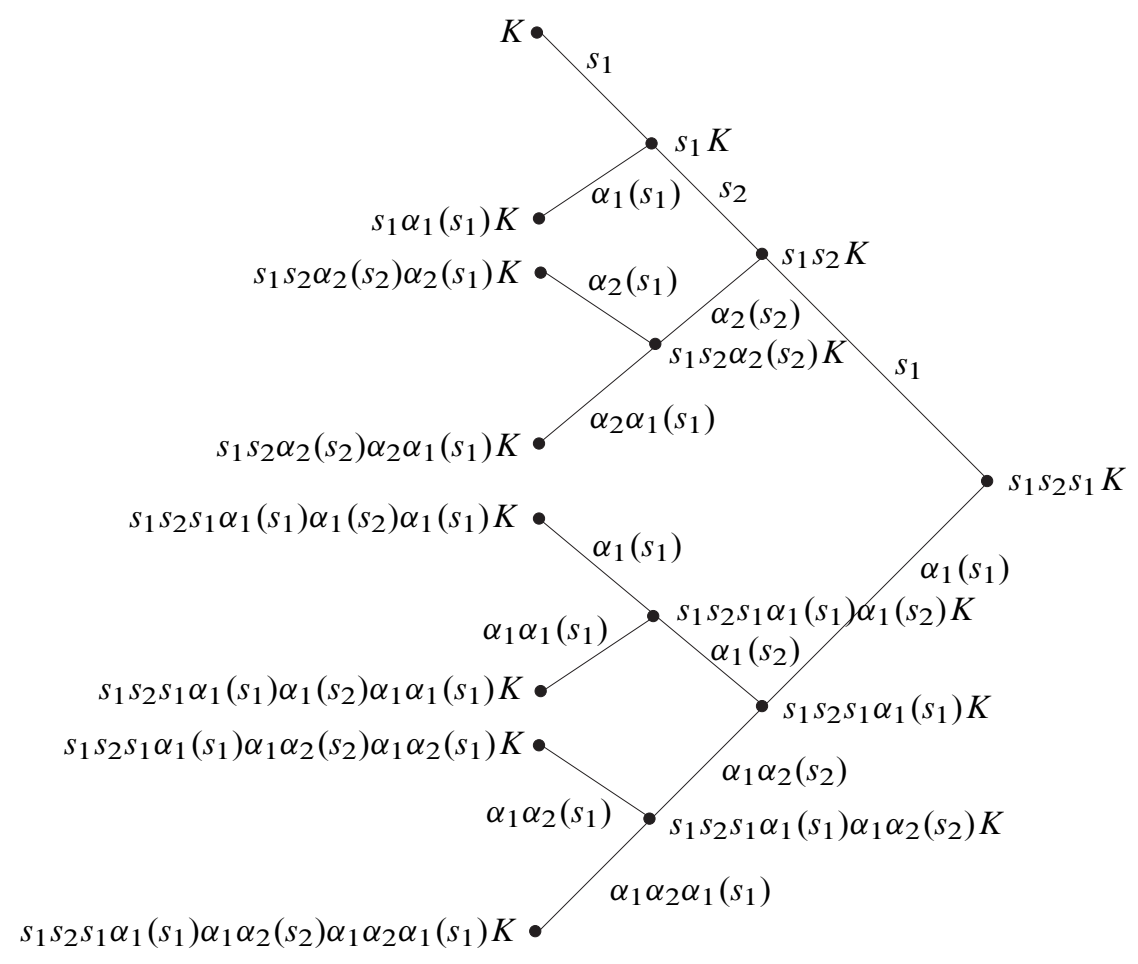

Figure 7. Dual graph for $Y_{4}$, with vertices and edges labelled.

Example. Let $(W, S), \alpha_{1}$ and $\alpha_{2}$ be as in the previous example of this section. To indicate the construction of $Y_{n}$ and $Y_{\infty}$ in this case, Figure 7 below depicts the dual graph for $Y_{4}$, that is, the graph with vertices the chambers of $Y_{4}$, and edges joining adjacent chambers. The edges are labelled with the type of the corresponding interior mirror. Figure 8 sketches the dual graph for $Y_{\infty}$.

We now describe features of $Y_{n}$ and $Y_{\infty}$ which will be needed below. The first lemma follows from the construction of $Y_{n}$ and $Y_{\infty}$ and Lemma 4.1 above.

Lemma 4.2. Let $w=w_{j_{n-1}, \ldots, j_{k}} \in W_{k, n}$. All of the chambers of $Y_{n}$ to which $w K \in Y_{k, n}$ is adjacent are described by the following.

(1) For $n \geq 1$ and $1 \leq k<n$, the chamber $w K$ is adjacent to exactly one chamber of $Y_{k+1, n}$, namely it is $\alpha^{j_{n-1}, \ldots, j_{k}}\left(s_{k}\right)$-adjacent to the chamber $w_{j_{n-1}, \ldots, j_{k+1}} K$ of $Y_{k+1, n}$.

(2) For $n \geq 2$ and $1 \leq k \leq n$, the chamber $w K$ is adjacent to exactly $q_{k-1}$ distinct chambers of $Y_{k-1, n}$, namely for each $0 \leq j_{k-1}<q_{k-1}$, the chamber $w K$ is

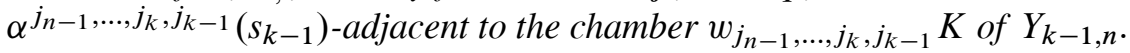

Similarly for $Y_{\infty}$. 


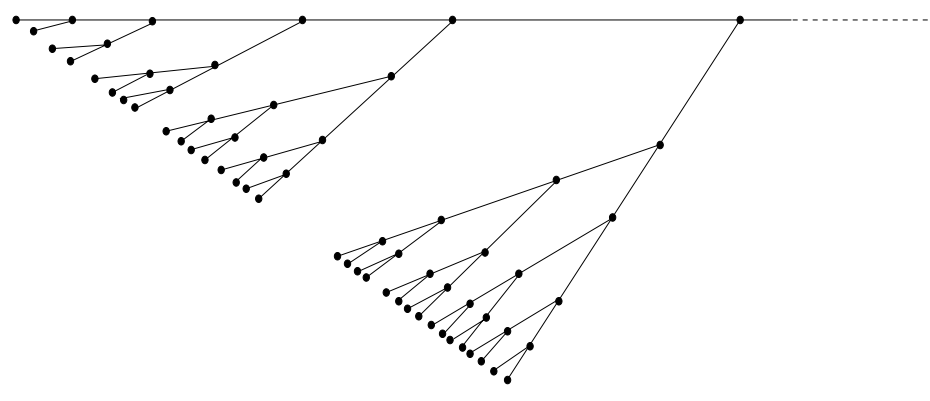

Figure 8. Dual graph for $Y_{\infty}$.

Corollary 4.3. (1) Any vertex of $Y_{n}$ is contained in at most two distinct chambers of $Y_{n}$, and similarly for $Y_{\infty}$.

(2) Any two interior mirrors of $Y_{n}$ or $Y_{\infty}$ are disjoint.

Proof. Suppose $\sigma$ is a vertex of $Y_{n}$, contained in the chamber $w K$, where $w$ is as in Lemma 4.2 above. If $\sigma$ is contained in more than one chamber of $Y_{n}$ or $Y_{\infty}$, then $\sigma$ is contained in an interior mirror $K_{s}$, for some $s \in S$. By the construction of $Y_{n}$ and Lemma 4.2 above, $s$ is either an image of $s_{k}$, or one of $q_{k-1}$ distinct images of $s_{k-1}$, under some element of $A$. Suppose $s$ is in the image of $s_{k}$. Condition (1) of the Main Theorem implies that $m_{s_{k} s_{k-1}}=\infty$. Hence the mirror $K_{s}$ is disjoint from each of the $q_{k-1}$ mirrors of types the $q_{k-1}$ images of $s_{k-1}$. Therefore the only chambers of $Y_{n}$ which contain $\sigma$ are the two chambers $w K$ and $w s K$. Now suppose that $s$ is one of the $q_{k-1}$ images of $s_{k-1}$ under some element of $A$. Condition (3) of the Main Theorem implies that the mirrors of types each of these images are pairwise disjoint, and so again $\sigma$ is contained in only two distinct chambers of $Y_{n}$. Similarly, any two interior mirrors of $Y_{n}$ or $Y_{\infty}$ are disjoint.

Corollary 4.4. For all $n \geq 2$, there are $q_{n-1}$ disjoint subcomplexes of $Y_{n}$, denoted by $Y_{n-1}^{j_{n-1}}$ for $0 \leq j_{n-1}<q_{n-1}$, each isomorphic to $Y_{n-1}$, and with $Y_{n-1}^{0}=Y_{n-1} \subset Y_{n}$. For each $0 \leq j_{n-1}<q_{n-1}$, the subcomplex $Y_{n-1}^{j_{n-1}}$ is attached to the chamber $w_{n} K=s_{1} s_{2} \ldots s_{n-1} K$ of $Y_{n}$ along its mirror of type $\alpha^{j_{n-1}}\left(s_{n-1}\right)$. An isomorphism

$$
F^{j_{n-1}}: Y_{n-1} \rightarrow Y_{n-1}^{j_{n-1}}
$$

is given by sending the chamber

$$
w_{j_{n-2}, \ldots, j_{k}} K \in Y_{k, n-1}
$$

to the chamber

$$
w_{j_{n-1}, j_{n-2}, \ldots, j_{k}} K \in Y_{k, n},
$$

and the vertex of $w_{j_{n-2}, \ldots, j_{k}} K$ of type $T$ to the vertex of $w_{j_{n-1}, j_{n-2}, \ldots, j_{k}} K$ of type $\alpha^{j_{n-1}}(T)$, for each spherical subset $T$ of $S$. 
Proof. Induction on $n$, using Lemma 4.2 and Corollary 4.3 above.

4.2. Complexes of groups $G\left(Y_{n}\right)$ and $G\left(Y_{\infty}\right)$. We now construct complexes of groups $G\left(Y_{n}\right)$ over each $Y_{n}$, and $G\left(Y_{\infty}\right)$ over $Y_{\infty}$, and show that there are coverings $G\left(Y_{n}\right) \rightarrow G\left(Y_{1}\right)$ and $G\left(Y_{\infty}\right) \rightarrow G\left(Y_{1}\right)$. To simplify notation, write $Y$ for $Y_{n}$ or $Y_{\infty}$.

To define the local groups of $G(Y)$, let $\sigma$ be a vertex of $Y$, of type $T$. By Corollary 4.3 above, $\sigma$ is contained in at most two distinct chambers of $Y$. If $\sigma$ is only contained in one chamber of $Y$, put $G_{\sigma}=W_{T}$. If $\sigma$ is contained in two distinct chambers of $Y$, then by Corollary 4.3 above $\sigma$ is contained in a unique interior mirror $K_{s}$, with $s \in T$. By the construction of $Y, s$ is in the $A$-orbit of some $s_{n}, n \geq 1$. By condition (4) of the Main Theorem, it follows that the group $W_{T}$ is halvable along $s$. We define the local group at $\sigma$ to be $G_{\sigma}=$ half $_{s}\left(W_{T}\right)$.

The monomorphisms between local groups are defined as follows. Let $a$ be an edge of $Y$, with $i(a)$ of type $T$ and $t(a)$ of type $T^{\prime}$, so that $T \subsetneq T^{\prime}$. If both of the vertices $i(a)$ and $t(a)$ are contained in a unique chamber of $Y$, then the monomorphism $\psi_{a}$ along this edge is defined to be the natural inclusion $W_{T} \hookrightarrow W_{T^{\prime}}$. If $i(a)$ is contained in two distinct chambers, then $i(a)$ is contained in a unique interior mirror $K_{s}$, with $s \in T$. Thus $s \in T^{\prime}$ as well, and so $t(a)$ is also contained in the mirror $K_{s}$. From the definitions of half ${ }_{s}\left(W_{T}\right)$ and half $_{s}\left(W_{T^{\prime}}\right)$, it follows that there is a natural inclusion half $f_{s}\left(W_{T}\right) \hookrightarrow \operatorname{half}_{s}\left(W_{T^{\prime}}\right)$, and we define $\psi_{a}$ be this inclusion. Finally suppose that $i(a)$ is contained in a unique chamber of $Y$ but $t(a)$ is contained in two distinct chambers of $Y$. Then for some $k \geq 1, i(a)$ is in a chamber of $Y_{k, n}$ (respectively, $Y_{k, \infty}$ ), and $t(a)$ is either in $Y_{k-1, n}$ or in $Y_{k+1, n}$ (respectively, in $Y_{k-1, \infty}$ or $\left.Y_{k+1, \infty}\right)$. Moreover $t(a)$ is contained in a unique interior mirror $K_{s}$, with $s \in T^{\prime}-T$. If $t(a)$ is in $Y_{k-1, n}$ (respectively, $Y_{k-1, \infty}$ ), then we define $\psi_{a}$ to be the natural inclusion $W_{T} \hookrightarrow \operatorname{half}_{s}\left(W_{T^{\prime}}\right)$. If $t(a)$ is in $Y_{k+1, n}$ (respectively, $Y_{k+1, \infty}$ ), then we define $\psi_{a}$ to be the monomorphism defined on the generators $t \in T$ of $W_{T}$ by $\psi_{a}(t):=s t s \in \operatorname{half}_{s}\left(W_{T^{\prime}}\right)$, that is, $\psi_{a}=\operatorname{Ad}(s)$.

It is not hard to verify that for all pairs of composable edges $(a, b)$ in $Y, \psi_{a b}=$ $\psi_{a} \circ \psi_{b}$. Hence we have constructed simple complexes of groups $G\left(Y_{n}\right)$ and $G\left(Y_{\infty}\right)$ over $Y_{n}$ and $Y_{\infty}$ respectively. Note that these complexes of groups are faithful, since by construction the local group at each vertex of type $\varnothing$ is trivial. Note also that $G\left(Y_{1}\right)$ is the same complex of groups as constructed in Section 2.3 above, which has fundamental group $W$ and universal cover $\Sigma$.

Example. Let $(W, S), \alpha_{1}$ and $\alpha_{2}$ be as in the examples in Section 4.1 above. The complex of groups $G\left(Y_{2}\right)$ is sketched in Figure 9. From left to right, the three chambers here are $K, s_{1} K$ and $s_{1} \alpha_{1}\left(s_{1}\right) K$. We denote by $D_{2 m}$ the dihedral group of order $2 m$, with $D_{m}$ the dihedral group of order $m$, and similarly for $D_{2 m^{\prime}}$ and $D_{m^{\prime}}$ (recall that $m$ and $m^{\prime}$ are even).

Proposition 4.5. There are coverings of complexes of groups $G\left(Y_{n}\right) \rightarrow G\left(Y_{1}\right)$ and $G\left(Y_{\infty}\right) \rightarrow G\left(Y_{1}\right)$. 


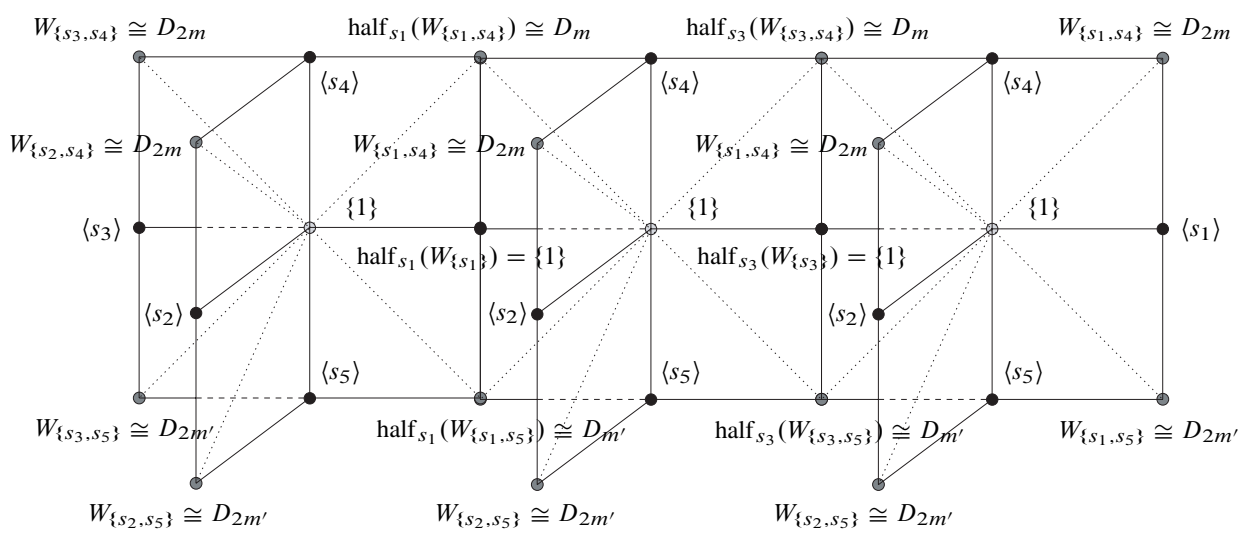

Figure 9. Complex of groups $G\left(Y_{2}\right)$.

Proof. Let $f_{n}: Y_{n} \rightarrow Y_{1}$ and $f_{\infty}: Y_{\infty} \rightarrow Y_{1}$ be the maps sending each vertex of $Y_{n}$ or $Y_{\infty}$ of type $T$ to the unique vertex of $Y_{1}=K$ of type $T$. Then by construction of $Y_{n}$ and $Y_{\infty}$, the maps $f_{n}$ and $f_{\infty}$ are nondegenerate morphisms of scwols. We define coverings $\Phi_{n}: G\left(Y_{n}\right) \rightarrow G\left(Y_{1}\right)$ and $\Phi_{\infty}: G\left(Y_{\infty}\right) \rightarrow G\left(Y_{1}\right)$ over $f_{n}$ and $f_{\infty}$ respectively. To simplify notation, write $Y$ for respectively $Y_{n}$ or $Y_{\infty}, f$ for respectively $f_{n}$ or $f_{\infty}$, and $\Phi$ for respectively $\Phi_{n}$ or $\Phi_{\infty}$.

Let $\sigma$ be a vertex of $Y$, of type $T$. If the local group at $\sigma$ is $G_{\sigma}=W_{T}$ then the map of local groups $\phi_{\sigma}: G_{\sigma} \rightarrow W_{T}$ is the identity map. If the local group at $\sigma$ is half $_{s}\left(W_{T}\right)$, for some $s \in T$, then $\phi_{\sigma}$ : half ${ }_{s}\left(W_{T}\right) \rightarrow W_{T}$ is the natural inclusion as an index 2 subgroup. To define elements $\phi(a)$, if the monomorphism $\psi_{a}$ in $G(Y)$ is natural inclusion, define $\phi(a)=1$. If $\psi_{a}$ is $\operatorname{Ad}(s)$, then define $\phi(a)=s$. It is then easy to check that, by construction, $\Phi$ is a morphism of complexes of groups.

To show that $\Phi$ is a covering of complexes of groups, we first observe that each of the local maps $\phi_{\sigma}$ is injective. Now fix $\sigma$ a vertex of $Y$, of type $T^{\prime}$, and $b$ an edge of $Y_{1}=K$ such that $t(b)=f(\sigma)$, with $i(b)$ of type $T$ (hence $T \subsetneq T^{\prime}$ ). We must show that the map

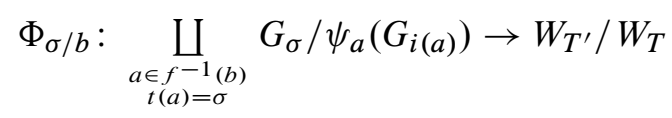

induced by $g \mapsto \phi_{\sigma}(g) \phi(a)$ is a bijection, where $G_{\sigma}$ and $G_{i(a)}$ are the local groups of $G(Y)$.

First suppose that $\sigma$ is contained in a unique chamber of $Y$. Then by construction, there is a unique edge $a$ of $Y$ with $i(a)$ of type $T$ and $t(a)=\sigma$, hence a unique edge $a \in f^{-1}(b)$ with $t(a)=\sigma$. Moreover, $G_{\sigma}=W_{T^{\prime}}, G_{i(a)}=W_{T}$, the monomorphism $\psi_{a}$ is natural inclusion hence $\phi(a)=1$, and $\phi_{\sigma}: G_{\sigma} \rightarrow W_{T^{\prime}}$ is the identity map. Hence $\Phi_{\sigma / b}$ is a bijection in this case. 
Now suppose that $\sigma$ is contained in two distinct chambers of $Y$. Then $\sigma$ is contained in a unique interior mirror $K_{s}$ of $Y$, with $s \in T^{\prime}$. Assume first that $s \in T$ as well. Then there is a unique edge $a$ of $Y$ with $i(a)$ of type $T$ and $t(a)=\sigma$. This edge is also contained in the mirror $K_{s}$. Hence there is a unique $a \in f^{-1}(b)$ with $t(a)=\sigma$. By construction, we have $G_{\sigma}=$ half $_{s}\left(W_{T^{\prime}}\right)$, the map $\phi_{\sigma}: G_{\sigma} \rightarrow W_{T^{\prime}}$ is natural inclusion as an index 2 subgroup, $G_{i(a)}=\operatorname{half}_{s}\left(W_{T}\right)$, the map $\psi_{a}$ is natural inclusion, and $\phi(a)$ trivial. Since the index $\left[W_{T^{\prime}}: W_{T}\right]=\left[\operatorname{half}_{s}\left(W_{T^{\prime}}\right): \operatorname{half}_{s}\left(W_{T}\right)\right]$ is finite, it is enough to verify that the inclusion half ${ }_{s}\left(W_{T^{\prime}}\right) \rightarrow W_{T^{\prime}}$ induces an injective map on cosets

$$
\operatorname{half}_{s}\left(W_{T^{\prime}}\right) / \operatorname{half}_{s}\left(W_{T}\right) \rightarrow W_{T^{\prime}} / W_{T} .
$$

For this, suppose that $w, w^{\prime} \in \operatorname{half}_{s}\left(W_{T^{\prime}}\right)$ and that $w W_{T}=w^{\prime} W_{T}$ in $W_{T^{\prime}}$. Then $w^{-1} w^{\prime} \in W_{T} \cap \operatorname{half}_{s}\left(W_{T^{\prime}}\right)$. By definitions, it follows that $w^{-1} w^{\prime} \in \operatorname{half}_{s}\left(W_{T}\right)$, as required.

Now assume that $\sigma$ is contained in the interior mirror $K_{s}$, with $s \notin T$. There are then two edges $a_{1}, a_{2} \in f^{-1}(b)$ such that $t\left(a_{1}\right)=t\left(a_{2}\right)=\sigma$. Without loss of generality, $\psi_{a_{1}}$ is natural inclusion $W_{T} \rightarrow$ half $_{s}\left(W_{T^{\prime}}\right)$ and $\phi\left(a_{1}\right)=1$, while $\psi_{a_{2}}(g)=s g s$ with $\phi\left(a_{2}\right)=s$. Since the index $\left[\operatorname{half}_{s}\left(W_{T^{\prime}}\right): W_{T}\right]=\frac{1}{2}\left[W_{T^{\prime}}: W_{T}\right]$ is finite, it is enough to show that the map on cosets $\Phi_{\sigma / b}$ is surjective. Let $w \in W_{T^{\prime}}$. If $w \in$ half $_{s}\left(W_{T^{\prime}}\right) \leq W_{T^{\prime}}$, then the image of the coset $w \psi_{a_{1}}\left(G_{i\left(a_{1}\right)}\right)=w W_{T}$ in $G_{\sigma}$ is the coset $w W_{T}$ in $W_{T^{\prime}}$. If $w \notin$ half $_{s}\left(W_{T^{\prime}}\right)$, then since half ${ }_{s}\left(W_{T^{\prime}}\right)$ has index 2 in $W_{T^{\prime}}$, and $s \notin$ half $_{s}\left(W_{T^{\prime}}\right)$, there is a $w^{\prime} \in$ half $_{s}\left(W_{T^{\prime}}\right) \leq W_{T^{\prime}}$ such that $w=w^{\prime} s$. The image of the coset $w^{\prime} \psi_{a_{2}}\left(G_{i\left(a_{2}\right)}\right)=w^{\prime}\left(s W_{T} s\right)$ in $\operatorname{half}_{s}\left(W_{T^{\prime}}\right)$ is then the coset $w^{\prime} \phi\left(a_{2}\right) W_{T}=w^{\prime} s W_{T}=w W_{T}$ in $W_{T^{\prime}}$. Thus $\Phi_{\sigma / b}$ is surjective, as required.

We conclude that $\Phi$ is a covering of complexes of groups.

4.3. Group actions on $\boldsymbol{Y}_{\boldsymbol{n}}$ and $\boldsymbol{Y}_{\infty}$. In this section we construct the action of a finite group $H_{n}$ on $Y_{n}$ in the sense of Definition 2.4 above, and that of an infinite group $H_{\infty}$ on $Y_{\infty}$.

We first define the groups $H_{n}$ and $H_{\infty}$. For each $n \geq 1$, let $C_{q_{n}}$ denote the cyclic group of order $q_{n}$. Note that $C_{q_{n}} \cong\left\langle\alpha_{n}\right\rangle$. We define $H_{1}$ to be the trivial group and $H_{2}=C_{q_{1}}$. For $n \geq 3$, we define $H_{n}$ to be the wreath product

$$
H_{n}=H_{n-1} \prec C_{q_{n-1}}=\left(\ldots\left(\left(C_{q_{1}} \prec C_{q_{2}}\right) \curlywedge C_{q_{3}}\right) \curlywedge \ldots\right) \prec C_{q_{n-1}}=C_{q_{1}} \prec C_{q_{2}} \prec \cdots \prec C_{q_{n-1}},
$$

that is, $H_{n}$ is the semidirect product by $C_{q_{n-1}}$ of the direct product of $q_{n-1}$ copies of $H_{n-1}$, where $C_{q_{n-1}}$ acts on this direct product by cyclic permutation of coordinates. Note that $H_{n}$ is a finite group of order

$$
\left|H_{n}\right|=q_{1}^{q_{2} q_{3} \ldots q_{n-1}} q_{2}^{q_{3} \ldots q_{n-1}} \ldots q_{n-2}^{q_{n-1}} q_{n-1} .
$$

We define $H_{\infty}$ to be the infinite iterated (unrestricted) wreath product

$$
H_{\infty}:=C_{q_{1}} \prec C_{q_{2}} \prec \cdots \prec C_{q_{n-1}} \prec \cdots
$$


We then have natural inclusions

$$
H_{1}<H_{2}<\cdots<H_{n}<\cdots<H_{\infty}
$$

The following lemma will be needed for the proof of Corollary 1.2 in Section 4.6 below.

Lemma 4.6. The group $H_{\infty}$ is not finitely generated.

Proof. By definition of $H_{\infty}$, for any nontrivial $h \in H_{\infty}$ there is an $n \geq 1$ such that $h \in H_{n}$.

We now define the actions of $H_{n}$ and $H_{\infty}$ on $Y_{n}$ and $Y_{\infty}$ respectively. This uses the label-preserving automorphisms $\alpha_{n} \in A$. Note that the action of $A$ on the nerve $L$ extends to the chamber $K$, fixing the vertex of type $\varnothing$. This action does not in general have a strict fundamental domain. Inconveniently, this action also does not satisfy condition (2) of Definition 2.4 above, since for any nontrivial $\alpha \in A$, there is an edge $a$ of $K$ with $i(a)$ of type $\emptyset$ but $\alpha(a) \neq a$. However, to satisfy Definition 2.4, it suffices to define actions on $Y_{n}$ and $Y_{\infty}$, and then extend in the obvious way to the scwols which are the barycentric subdivisions of these spaces, with naturally oriented edges.

For each $n \geq 1$ fix a generator $a_{n}$ for the cyclic group $C_{q_{n}}$. Recall that $\alpha_{n} \in A$ has order $q_{n}$. Thus for any $\alpha \in A$, there is a faithful representation $C_{q_{n}} \rightarrow A$, given by $a_{n} \mapsto \alpha \alpha_{n} \alpha^{-1}$. Recall also that $\alpha_{n}$ fixes the star in $L$ of the vertex $s_{n+1}$, and that $\left\langle\alpha_{n}\right\rangle$ acts freely on the $\left\langle\alpha_{n}\right\rangle$-orbit of $s_{n}$. Hence $a_{n} \mapsto \alpha \alpha_{n} \alpha^{-1}$ induces an action of $C_{q_{n}}$ on the chamber $K$, which fixes pointwise the mirror of type $\alpha\left(s_{n+1}\right)$, and permutes cyclically the set of mirrors of types $\alpha \alpha_{n}^{j_{n}}\left(s_{n}\right)$, for $0 \leq j_{n}<q_{n}$.

We define the action of $H_{n}$ on $Y_{n}$ inductively as follows. The group $H_{1}$ is trivial. For $n \geq 2$, assume that the action of $H_{n-1}$ on $Y_{n-1}$ has been given. The subgroup $C_{q_{n-1}}$ of $H_{n}$ then fixes the chamber $w_{n} K=s_{1} s_{2} \ldots s_{n-1} K$ of $Y_{n}$ setwise, and acts on this chamber via $a_{n-1} \mapsto \alpha_{n-1}$. By the discussion above, this action fixes pointwise the mirror of type $s_{n}$ of $w_{n} K$, and permutes cyclically the $q_{n-1}$ mirrors of types $\alpha_{n-1}^{j_{n-1}}\left(s_{n-1}\right)$, with $0 \leq j_{n-1}<q_{n-1}$, along which (by Lemma 4.4 above), $q_{n-1}$ disjoint subcomplexes of $Y_{n}$, each isomorphic to $Y_{n-1}$, are attached.

By induction, a copy of $H_{n-1}$ in $H_{n}$ acts on each of these copies of $Y_{n-1}$ in $Y_{n}$. More precisely, for $0 \leq j_{n-1}<q_{n-1}$, the $j_{n-1}$ st copy of $H_{n-1}$ in $H_{n}$ acts on the subcomplex $Y_{n-1}^{j_{n-1}}$ of Lemma 4.4 above. This action is given by conjugating the (inductively defined) action of $H_{n-1}$ on $Y_{n-1} \subset Y_{n}$ by the isomorphism $F^{j_{n-1}}: Y_{n-1} \rightarrow Y_{n-1}^{j_{n-1}}$ in Lemma 4.4. By definition, the action of $C_{q_{n-1}}$ cyclically permutes the subcomplexes $Y_{n-1}^{j_{n-1}}$, and so we have defined an action of $H_{n}$ on $Y_{n}$. The action of $H_{\infty}$ on $Y_{\infty}$ is similar.

We now describe the fundamental domains for these actions. For each $n \geq 1$ and each $1 \leq k \leq n$, observe that $H_{n}$ acts transitively on the set of chambers 
$Y_{k, n}$. Let $K_{1}=K$, and for $n \geq 2$ let $K_{n}$ be the quotient of the chamber $w_{n} K=$ $s_{1} s_{2} \ldots s_{n-1} K$ by the action of $C_{q_{n-1}} \leq H_{n}$ as defined above. In $K_{n}$, the mirrors of types $\alpha_{n-1}^{j_{n-1}}\left(s_{n-1}\right)$, for $0 \leq j_{n-1}<q_{n-1}$, have been identified. By abuse of notation, we refer to these identified mirrors as the mirror of type $s_{n-1}$ of $K_{n}$. Note also that $C_{q_{n-1}} \leq H_{n}$ fixes pointwise the mirror of type $s_{n}$ of $w_{n} K$, and so we may speak of the mirror of type $s_{n}$ of $K_{n}$. Then a fundamental domain for the action of $H_{n}$ on $Y_{n}$ is the finite complex

$$
Z_{n}:=\left(K_{1} \cup K_{2} \cup \cdots \cup K_{n}\right) / \sim,
$$

where $\sim$ means we identify the $s_{i-1}$-mirrors of $K_{i-1}$ and $K_{i}$, for $1 \leq i<n$. Similarly, a fundamental domain for the action of $H_{\infty}$ on $Y_{\infty}$ is the infinite complex

$$
Z_{\infty}:=\left(K_{1} \cup K_{2} \cup \cdots \cup K_{n} \cup \cdots\right) / \sim .
$$

Finally we describe the stabilisers in $H_{n}$ and $H_{\infty}$ of the vertices of $Y_{n}$ and $Y_{\infty}$. Let $w K$ be a chamber of $Y_{n}$ or $Y_{\infty}$. Then there is a smallest $k \geq 1$ such that $w K \in Y_{k}$. By construction, it follows that the stabiliser in $H_{n}$ or $H_{\infty}$ of any vertex in the chamber $w K$ is a subgroup of the finite group $H_{k}$. Hence $H_{n}$ and $H_{\infty}$ act with finite stabilisers. Note also that for every $n \geq 1$, the action of $H_{n}$ fixes the vertex of type $\emptyset$ in the chamber $w_{n} K$. We may thus speak of the vertex of type $\emptyset$ in the quotient $K_{n}$ defined above. In fact, in the fundamental domains $Z_{n}$ and $Z_{\infty}$ defined above, the vertex of type $\emptyset$ in $K_{n}$, for $n \geq 1$, has a lift in $Y_{n}$ or $Y_{\infty}$ with stabiliser the finite group $H_{n}$. We observe also that the actions of $H_{n}$ and $H_{\infty}$ are faithful, since the stabiliser of the vertex of type $\emptyset$ of $K_{1}=K$ is the trivial group $H_{1}$. Figure 10 shows $Z_{\infty}$ and the stabilisers of (lifts of) its vertices of type $\emptyset$ for the example in Section 4.1 above.

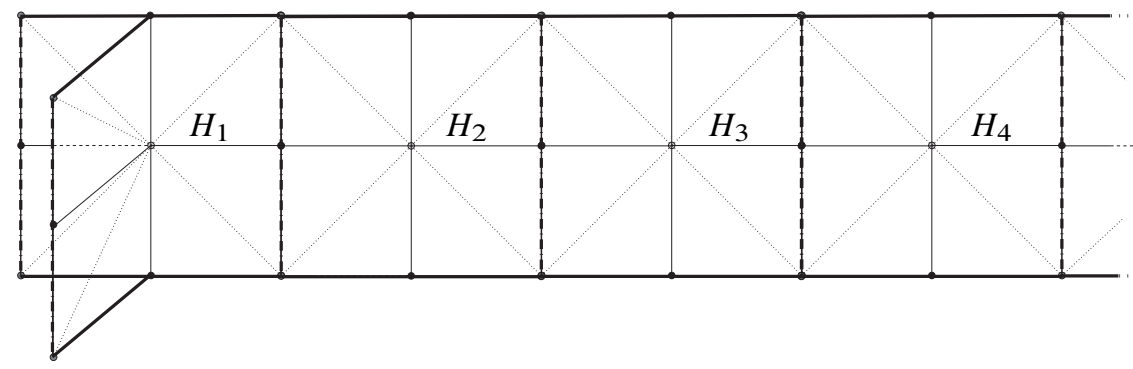

Figure 10. Fundamental domain $Z_{\infty}$.

4.4. Group actions on $\boldsymbol{G}\left(Y_{\boldsymbol{n}}\right)$ and $\boldsymbol{G}\left(\boldsymbol{Y}_{\infty}\right)$. In this section we show that the actions of $H_{n}$ and $H_{\infty}$ on $Y_{n}$ and $Y_{\infty}$, defined in Section 4.3 above, extend to actions (by simple morphisms) on the complexes of groups $G\left(Y_{n}\right)$ and $G\left(Y_{\infty}\right)$. To simplify notation, write $H$ for $H_{n}$ or $H_{\infty}, Y$ for $Y_{n}$ or $Y_{\infty}$, and $Z$ for $Z_{n}$ or $Z_{\infty}$. Technically, 
instead of working with $G(Y)$, we work with the corresponding naturally defined complex of groups over the barycentric subdivision of $Y$, so that the action of $H$ satisfies Definition 2.4 above. By abuse of notation we will however continue to write $G(Y)$.

Recall that for $\sigma$ a vertex of $Y$ of type $T$, the local group $G_{\sigma}$ is either $W_{T}$ or half $_{S}\left(W_{T}\right)$, and the latter occurs if and only if $\sigma$ is contained in an interior $s$-mirror of $Y$ with $s \in T$. Let $w K$ be a chamber of $Y$ and let $h \in H$. By definition of the $H$-action, there is an $\alpha \in A$ such that for each vertex $\sigma$ in $w K$, with $\sigma$ of type $T$, the vertex $h \cdot \sigma$ of $h \cdot w K$ has type $\alpha(T)$. Moreover, if $\sigma$ is contained in an interior $s$-mirror then $h \cdot \sigma$ is contained in an interior $\alpha(s)$-mirror. We may thus define the local $\operatorname{map} \phi_{\sigma}^{h}: G_{\sigma} \rightarrow G_{h \cdot \sigma}$ by $\phi_{\sigma}^{h}(t)=\alpha(t)$ for each $t \in T$, and (if $G_{\sigma}=\operatorname{half}_{s}\left(W_{T}\right)$ ), $\phi_{\sigma}^{h}(s t s)=\alpha(s) \alpha(t) \alpha(s)$. Then $\phi_{\sigma}^{h}$ is an isomorphism either $W_{T} \rightarrow W_{\alpha(T)}$, or half $_{s}\left(W_{T}\right) \rightarrow$ half $_{\alpha(s)}\left(W_{\alpha(T)}\right)$, as appropriate. It is not hard to verify that these local maps define an action of $H$ on $G(Y)$ by simple morphisms.

4.5. Conclusion. In this section we combine the results of Sections 4.1-4.4 above to complete the proof of the Main Theorem.

Recall that $G\left(Y_{1}\right)$ is developable with universal cover $\Sigma$ (see Section 2.3). By Proposition 3.9 and Theorem 3.2 above, it follows that the complexes of groups $G\left(Y_{n}\right)$ and $G\left(Y_{\infty}\right)$ are developable with universal cover $\Sigma$. Let $H\left(Z_{n}\right)$ be the complex of groups induced by $H_{n}$ acting on $G\left(Y_{n}\right)$, and $H\left(Z_{\infty}\right)$ that induced by $H_{\infty}$ acting on $G\left(Y_{\infty}\right)$. By Theorem 3.1 above, there are coverings of complexes of groups $G\left(Y_{n}\right) \rightarrow H\left(Z_{n}\right)$ and $G\left(Y_{\infty}\right) \rightarrow H\left(Z_{\infty}\right)$. Hence (by Theorem 3.2 above) each $H\left(Z_{n}\right)$ and $H\left(Z_{\infty}\right)$ is developable with universal cover $\Sigma$.

Let $\Gamma_{n}$ be the fundamental group of $H\left(Z_{n}\right)$ and $\Gamma$ the fundamental group of $H\left(Z_{\infty}\right)$. Since the complexes of groups $G\left(Y_{n}\right)$ and $G\left(Y_{\infty}\right)$ are faithful, and the actions of $H_{n}$ and $H_{\infty}$ are faithful, Theorem 3.1 above implies that $H\left(Z_{n}\right)$ and $H\left(Z_{\infty}\right)$ are faithful complexes of groups. Thus $\Gamma_{n}$ and $\Gamma$ may be identified with subgroups of $G=\operatorname{Aut}(\Sigma)$. Now $G\left(Y_{n}\right)$ and $G\left(Y_{\infty}\right)$ are complexes of finite groups, and the $H_{n}$ - and $H_{\infty}$-actions have finite vertex stabilisers. Hence by construction, $H\left(Z_{n}\right)$ and $H\left(Z_{\infty}\right)$ are complexes of finite groups. Therefore $\Gamma_{n}$ and $\Gamma$ are discrete subgroups of $G$. Since the fundamental domain $Z_{n}$ is finite, it follows that each $\Gamma_{n}$ is a uniform lattice. To show that $\Gamma$ is a nonuniform lattice, we use the normalisation of Haar measure $\mu$ on $G=\operatorname{Aut}(\Sigma)$ defined in Section 2.1 above, with the $G$-set $V$ the set of vertices of $\Sigma$ of type $\emptyset$. Since the local groups of $H\left(Z_{\infty}\right)$ at the vertices of type $\emptyset$ in $Z_{\infty}$ are $H_{1}, H_{2}, \ldots$, we have

$$
\mu(\Gamma \backslash G)=\sum_{n=1}^{\infty} \frac{1}{\left|H_{n}\right|} .
$$

This series converges (see equation (4) above for the order of $H_{n}$, and note that each $q_{n} \geq 2$ ). We conclude that $\Gamma$ is a nonuniform lattice in $G$. Moreover, as the 
covolumes of the uniform lattices $\Gamma_{n}$ are the partial sums of this series, we have $\mu\left(\Gamma_{n} \backslash G\right) \rightarrow \mu(\Gamma \backslash G)$, as required. This completes the proof of the Main Theorem.

4.6. Proof of Corollary 1.2. The nonuniform lattice $\Gamma$ is the fundamental group of the complex of groups $H\left(Z_{\infty}\right)$ induced by the action of $H_{\infty}$ on $G\left(Y_{\infty}\right)$. By the short exact sequence in Theorem 3.1 above, there is a surjective homomorphism $\Gamma \rightarrow H_{\infty}$. Since $H_{\infty}$ is not finitely generated (Lemma 4.6 above), we conclude that $\Gamma$ is not finitely generated.

\section{Examples}

In this section we describe several infinite families of examples to which the Main Theorem applies. By the dimension of the Davis complex $\Sigma$ for a Coxeter system $(W, S)$, we mean the maximum cardinality of a spherical subset of $S$. We note that there may be maximal spherical special subgroups $W_{T}$ with $|T|$ strictly less than $\operatorname{dim}(\Sigma)$.

5.1. Two-dimensional examples. If $\operatorname{dim}(\Sigma)=2$ then the nerve of the Coxeter system $(W, S)$ is a graph $L$ with vertex set $S$ and two vertices $s$ and $t$ joined by an edge if and only if $m_{s t}$ is finite. Assume for simplicity that for some integer $m \geq 2$ all finite $m_{s t}=m$. Then $\Sigma$ is the barycentric subdivision of a polygonal complex $X$, with all 2-cells of $X$ regular Euclidean $2 m$-gons, and the link of every vertex of $X$ the graph $L$. Such an $X$ is called a $(2 m, L)$-complex. Condition (4) of the Main Theorem can hold only if $m$ is even, and so we also assume this. It is then not hard to find graphs $L$ so that, for some pair $s_{1}$ and $s_{2}$ of non-adjacent vertices of $L$, and for some nontrivial elements $\alpha_{1}, \alpha_{2} \in \operatorname{Aut}(L)$, conditions (1), (2) and (3) of the Main Theorem also hold. We present three infinite families of examples.

5.1.1. Buildings with complete bipartite links. Let $L$ be the complete bipartite graph $K_{q, q^{\prime}}$, with $q, q^{\prime} \geq 2$. If $q \geq 3$ then there are (nonadjacent) vertices $s_{1}$ and $s_{2}$ of $L$, and nontrivial elements $\alpha_{1}$ and $\alpha_{2}$ of $\operatorname{Aut}(L)$, so that the Main Theorem applies.

If $m=2$ then $\Sigma$ is the barycentric subdivision of the product of trees $T_{q} \times T_{q^{\prime}}$, where $T_{q}$ is the $q$-regular tree. In particular, if $m=m^{\prime}=2$ in Example 1 of Section 2.2 above, then $\Sigma$ is the barycentric subdivision of $T_{3} \times T_{2}$. If $m \geq 4$, then by Theorem 12.6.1 of [11] the complex $\Sigma$ may be metrised as a piecewise hyperbolic CAT(-1) polygonal complex. With this metric, if $p=2 m$ and $q=q^{\prime}$ then $\Sigma$ is the barycentric subdivision of Bourdon's building $I_{p, q}$ (studied in, for example, [6] and [7]), which is the unique 2-complex with all 2-cells regular right-angled hyperbolic $p$-gons $P$, and the link of every vertex the complete bipartite graph $K_{q, q}$. Bourdon's building is a right-angled hyperbolic building, of type $\left(W^{\prime}, S^{\prime}\right)$ where $W^{\prime}$ is the Coxeter group generated by the set of reflections $S^{\prime}$ in the sides of $P$. 
5.1.2. Fuchsian buildings. A Fuchsian building is a 2-dimensional hyperbolic building. Bourdon's building $I_{p, q}$ is a (right-angled) Fuchsian building. For Fuchsian buildings which are not right-angled see, for example, [5] and [13].

To show that the Main Theorem applies to certain Fuchsian buildings which are not right-angled, let $L$ be the finite building of rank 2 associated to a Chevalley group $\mathcal{E}$ (see [25]). Then $L$ is a bipartite graph, with vertex set say $S=S_{1} \sqcup S_{2}$, and for some $k \in\{3,4,6,8\}, L$ has girth $2 k$ and diameter $k$. Figure 11 depicts the building $L$ for the group $\mathcal{E}=\operatorname{GL}\left(3, \mathbb{F}_{2}\right)=\operatorname{GL}(3,2)$, for which $k=3$. The white vertices of this building may be identified with the set of one-dimensional subspaces of the vector space $V=\mathbb{F}_{2} \times \mathbb{F}_{2} \times \mathbb{F}_{2}$, and the black vertices with the set of two-dimensional subspaces of $V$. Two vertices are joined by an edge if those two subspaces are incident.

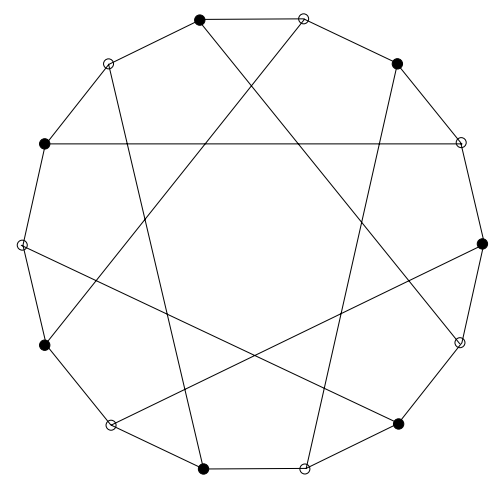

Figure 11. The building $L$ for $\mathcal{G}=\operatorname{GL}(3,2)$.

The group $\mathcal{E}$ acts on $L$, preserving the type of vertices, with quotient an edge. Suppose $s_{1} \in S_{1}$, and let $s_{2} \in S_{2}$ be a vertex at distance $k$ from $s_{1}$. Since $L$ is a thick building, there is more than one such vertex $s_{2}$. For $i=1,2$, the stabiliser $P_{i}$ of $s_{i}$ in $\mathcal{E}$ acts transitively on the set of vertices of $L$ at distance $k$ from $s_{i}$. Now, by Theorem 6.18 of [25], $P_{i}$ has a Levi decomposition

$$
P_{i}=U_{i} \rtimes L_{i}
$$

where $L_{i}$ is the subgroup of $P_{i}$ fixing the vertex $s_{3-i}$. Moreover, by Lemma 6.5 of [25], $U_{i}$ fixes the star of $s_{i}$ in $L$. Hence we may find elements $\alpha_{3-i} \in U_{i}$ for which conditions (1) and (2) of the Main Theorem hold. Condition (3) of the Main Theorem follows since $L$ is bipartite and the action of $\mathcal{E}$ preserves the type of vertices. For example, for $L$ as in Figure 11, if $s_{1}$ is the vertex $\{(1,0,0)\}$, we may choose $s_{2}$ to be the vertex $\{(0,1,0),(0,0,1),(0,1,1)\}$, and then choose

$$
\alpha_{1}=\left(\begin{array}{lll}
1 & 0 & 0 \\
1 & 1 & 0 \\
1 & 0 & 1
\end{array}\right) \quad \text { and } \quad \alpha_{2}=\left(\begin{array}{lll}
1 & 1 & 1 \\
0 & 1 & 0 \\
0 & 0 & 1
\end{array}\right)
$$


Suppose now that $L$ as above is the nerve of a Coxeter system $(W, S)$. By Theorem 12.6.1 of [11], since $L$ has girth $\geq 6$, the corresponding Davis complex $\Sigma$ may also be metrised as a piecewise hyperbolic CAT $(-1)$ polygonal complex. With this metrisation, $\Sigma$ is then the barycentric subdivision of a Fuchsian building, with the link of every vertex $L$ and all 2-cells regular hyperbolic $2 m$-gons (of vertex angle $\left.\frac{\pi}{k}\right)$. We call such a building a $(2 m, L)$-building. In general, there may be uncountably many isomorphism classes of (2m, $L$ )-buildings (see for instance [13]). In fact, the Davis complex $\Sigma$ is the barycentric subdivision of the unique locally reflexive $(2 m, L)$-building with trivial holonomy (see Haglund [15]).

5.1.3. Platonic polygonal complexes. A polygonal complex $X$ is Platonic if $\operatorname{Aut}(X)$ acts transitively on the set of flags (vertex, edge, face) in $X$. Any Platonic polygonal complex is a $(k, L)$-complex, with $k \geq 3$ and $L$ a graph such that $\operatorname{Aut}(L)$ acts transitively on the set of oriented edges in $L$. In [27], Świątkowski studied CAT(0) Platonic polygonal complexes $X$, where $L$ is a trivalent graph. Such complexes are not in general buildings.

A graph $L$ is said to be $n$-arc regular, for some $n \geq 1$, if $\operatorname{Aut}(L)$ acts simply transitively on the set of edge paths of length $n$ in $L$. For example, the Petersen graph in Figure 1 above is 3-arc regular. Any finite, connected, trivalent graph $L$, with $\operatorname{Aut}(L)$ transitive on the set of oriented edges of $L$, is $n$-arc regular for some $n \in\{1,2,3,4,5\}$ (Tutte [31]). Świątkowski [27] showed that if $n \in\{3,4,5\}$, then for all $k \geq 4$ there is a unique $(k, L)$-complex $X$, with $X$ Platonic. Thus if $k=2 m$ is even, the barycentric subdivision of $X$ is the Davis complex $\Sigma$ for $(W, S)$, where $(W, S)$ has nerve $L$ and all finite $m_{s t}=m$.

Now suppose that $L$ is a finite, connected, trivalent, $n$-arc regular graph with $n \in\{3,4,5\}$. Choose vertices $s_{1}$ and $s_{2}$ of $L$ at distance two in $L$ if $n=3,4$, and at distance three in $L$ if $n=5$. Then by Propositions 3-5 of Djoković-Miller [12], for $i=1,2$ there are involutions $\alpha_{i} \in \operatorname{Aut}(L)$ such that $\alpha_{i}$ fixes the star of $s_{3-i}$ in $L$, and $\alpha_{i}\left(s_{i}\right) \neq s_{i}$ is not adjacent to $s_{i}$. Thus if $m$ is even, the Main Theorem applies to $G=\operatorname{Aut}(\Sigma)$.

5.2. Higher-dimensional examples. We now discuss examples in dimension $>2$ to which the Main Theorem applies. The construction of the building $\Sigma$ below was suggested by an anonymous referee (our own examples were just for $W$ right-angled).

We first discuss when condition (4) in the Main Theorem can hold. Suppose $W_{T}$ is a spherical special subgroup of $W$, with $k=|T|>2$. If $W_{T}$ is irreducible, then from the classification of spherical Coxeter groups (see, for example, [11]), it is not hard to verify that $W_{T}$ is halvable along $s \in T$ if and only if $W_{T}$ is of type $B_{k}$, with $s \in T$ the unique generator so that $m_{s t} \in\{2,4\}$ for all $t \in T-\{s\}$; in this case half ${ }_{s}\left(W_{T}\right)$ is of type $D_{k}$. If $W_{T}$ is reducible, then as long as $s$ is contained in a direct factor $W_{T^{\prime}}, T^{\prime} \subsetneq T$, such that either $W_{T^{\prime}}=\langle s\rangle \cong C_{2}, W_{T^{\prime}}$ is an even dihedral group, or $W_{T^{\prime}}$ is of type $B_{j}$ with $j<k$ and $s$ the particular generator described above, then $W_{T}$ will be halvable along $s$. 
Now let $L$ be a thick spherical building of rank $k>2$. A reducible example is $L$ the join of $k$ sets of points, with each set having cardinality at least 3 . An irreducible example is $L$ the building for a Chevalley group $\mathcal{G}$ of rank $k$ over a finite field, such as $\operatorname{GL}(k+1,2)$.

Define a Coxeter group $W$ with nerve $L$ as follows. Fix $\Delta$ a chamber of $L$. Then $\Delta$ is a simplex on $k$ vertices. Let $p: L \rightarrow \Delta$ be the projection onto this chamber. Label the edges of $\Delta$ by the $m_{s t}$ for a finite Coxeter group $V$ on $k$ generators such that $V$ is a product of cyclic groups of order 2, even dihedral groups and copies of $B_{j}, j<k$. For example, when $V$ is right-angled all $m_{s t}=2$. Pull the edge labels of $\Delta$ back via $p$ to obtain a labelling of the edges of $L$. This defines a Coxeter group $W$ with nerve $L$, so that each maximal spherical special subgroup of $W$ is isomorphic to $V$.

The Davis complex $\Sigma$ for $W$ is tiled by copies of the barycentric subdivision of the Coxeter polytope $P$ associated to $V$. For example, when $V$ is right-angled, $P$ is a $k$-cube. The link of each vertex of $P$ is $L$. Applying the metric criterion of Charney-Lytchak [10], it follows that $\Sigma$ is the barycentric subdivision of a building. Note that $\operatorname{dim}(\Sigma)=k>2$.

Choose vertices $s_{1}$ and $s_{2}$ in $L$ which are opposite (see [25]). By the same arguments as in Section 5.1.2 above, there are (type-preserving) elements $\alpha_{1}, \alpha_{2} \in$ $\operatorname{Aut}(L)$ so that conditions (1)-(3) of the Main Theorem hold. A careful choice of $V$, such that $s_{1}$ and $s_{2}$ if contained in some copy of $B_{j}$ are both the required generators, then guarantees that condition (4) of the Main Theorem holds. Hence the Main Theorem applies to many examples of buildings of dimension $>2$.

We do not know of any hyperbolic buildings of dimension $>2$ to which the Main Theorem applies. For the 3-dimensional constructions of Haglund-Paulin in [20], certain of the $m_{s t}$ must be equal to 3, so condition (4) of the Main Theorem will not hold.

A slight modification of the above construction, for example by adding a vertex $s$ to $L$ with $m_{s t}=\infty$ for all $t \in S-\{s\}$, produces nerves which are not buildings, hence examples of $\Sigma$ of dimension $>2$ which are not buildings.

\section{References}

[1] W. Ballmann and M. Brin, Polygonal complexes and combinatorial group theory. Geom. Dedicata 50 (1994), 165-191. Zbl 0832.57002 MR 1279883

[2] H. Bass and R. Jiang, Automorphism groups of tree actions and of graphs of groups. $J$. Pure Appl. Algebra 112 (1996), 109-155. Zbl 0862.20021 MR 1402782

[3] H. Bass and A. Lubotzky, Tree lattices. Progr. Math. 176, Birkhäuser, Boston 2001. Zbl 1053.20026 MR 1794898

[4] A. Borel and G. Harder, Existence of discrete cocompact subgroups of reductive groups over local fields. J. Reine Angew. Math. 298 (1978), 53-64. Zbl 0385.14014 MR 483367 
[5] M. Bourdon, Sur les immeubles fuchsiens et leur type de quasi-isométrie. Ergodic Theory Dynam. Systems 20 (2000), 343-364. Zbl 0965.53030 MR 1756974

[6] M. Bourdon, Immeubles hyperboliques, dimension conforme et rigidité de Mostow. Geom. Funct. Anal. 7 (1997), 245-268. Zbl 0876.53020 MR 1445387

[7] M. Bourdon and H. Pajot, Rigidity of quasi-isometries for some hyperbolic buildings. Comment. Math. Helv. 75 (2000), 701-736. Zbl 0976.30011 MR 1789183

[8] M. R. Bridson and A. Haefliger, Metric spaces of non-positive curvature. Grundlehren Math. Wiss. 319, Springer-Verlag, Berlin 1999. Zbl 0988.53001 MR 1744486

[9] M. Burger and S. Mozes, Lattices in product of trees. Inst. Hautes Études Sci. Publ. Math. 92 (2000), 151-194. Zbl 1007.22013 MR 1839489

[10] R. Charney and A. Lytchak, Metric characterizations of spherical and Euclidean buildings. Geom. Topol. 5 (2001), 521-550. Zbl 1002.51008 MR 1833752

[11] M. W. Davis, The geometry and topology of Coxeter groups. London Math. Soc. Monogr. Ser. 32, Princeton University Press, Princeton, NJ, 2008. Zbl 1142.20020 MR 2360474

[12] D. Ž. Djoković and G. L. Miller, Regular groups of automorphisms of cubic graphs. $J$. Combin. Theory Ser. B 29 (1980), 195-230. Zbl 0385.05040 MR 586434

[13] D. Gaboriau and F. Paulin, Sur les immeubles hyperboliques. Geom. Dedicata 88 (2001), 153-197. Zbl 1020.51007 MR 1877215

[14] F. Haglund, Les polyèdres de Gromov. C. R. Acad. Sci. Paris Sér. I Math. 313 (1991), 603-606. Zbl 0749.52011 MR 1133493

[15] F. Haglund, Réseaux de Coxeter-Davis et commensurateurs. Ann. Inst. Fourier (Grenoble) 48 (1998), 649-666. Zbl 0899.20019 MR 1644077

[16] F. Haglund, Existence, unicité et homogénéité de certains immeubles hyperboliques. Math. Z. 242 (2002), 97-148. Zbl 1052.51009 MR 1985452

[17] F. Haglund, Commensurability and separability of quasiconvex subgroups. Algebr. Geom. Topol. 6 (2006), 949-1024. Zbl 1179.20038 MR 2240922

[18] F. Haglund, Finite index subgroups of graph products. Geom. Dedicata 135 (2008), 167-209. Zbl 1195.20047 MR 2413337

[19] F. Haglund and F. Paulin, Simplicité de groupes d'automorphismes d'espaces à courbure négative. In The Epstein birthday schrift, Geom. Topol. Monogr. 1, Geom. Topol. Publ., Coventry 1998, 181-248. Zbl 0916.51019 MR 1668359

[20] F. Haglund and F. Paulin, Constructions arborescentes d'immeubles. Math. Ann. 325 (2003), 137-164. Zbl 1025.51014 MR 1957268

[21] A. Kubena and A. Thomas, Density of commensurators for uniform lattices of rightangled buildings. J. Group Theory 15 (2012), 565--611.

[22] S. Lim and A. Thomas, Covering theory for complexes of groups. J. Pure Appl. Algebra 212 (2008), 1632-1663. Zbl 1182.57003 MR 2400734

[23] A. Lubotzky, Tree-lattices and lattices in Lie groups. In Combinatorial and geometric group theory, London Math. Soc. Lecture Note Ser. 204, Cambridge Univ. Press, Cambridge 1995, 217-232. Zbl 0840.22019 MR 1320284

[24] G. A. Margulis, Discrete subgroups of semisimple Lie groups. Springer-Verlag, New York 1991. Zbl 0732.22008 MR 1090825 
[25] M. Ronan, Lectures on buildings. Perspect. Math. 7, Academic Press, Boston 1989. Zbl 0694.51001 MR 1005533

[26] J.-P. Serre, Cohomologie des groupes discrets. In Prospects in mathematics, Ann. of Math. Stud. 70, Princeton Univ. Press, Princeton, N.J., 1971, 77-169. Zbl 0235.22020 MR 0385006

[27] J. Świątkowski, Trivalent polygonal complexes of nonpositive curvature and Platonic symmetry. Geom. Dedicata 70 (1998), 87-110. Zbl 0896.20028 MR 1612350

[28] T. Tamagawa, On discrete subgroups of $p$-adic algebraic groups. In Arithmetical Algebraic Geometry, Harper \& Row, New York 1965, 11-17. Zbl 0158.27801 MR 0195864

[29] A. Thomas, Lattices acting on right-angled buildings. Algebr. Geom. Topol. 6 (2006), 1215-1238. Zbl 1128.22002 MR 2253444

[30] A. Thomas, On the set of covolumes of lattices for Fuchsian buildings. C. R. Math. Acad. Sci. Paris 344 (2007), 215-218. Zbl 1150.20013 MR 2292989

[31] W. Tutte, A family of cubical graphs. Proc. Cambridge Philos. Soc. 43 (1947), 459-474. Zbl 0029.42401 MR 0021678

[32] G. White, Automorphisms of geometric structures associated to Coxeter groups. Preprint, arXiv:1202.6441 [math.GR].

[33] X. Xie, Quasi-isometric rigidity of Fuchsian buildings. Topology 45 (2006), 101-169. Zbl 1083.51008 MR 2170496

Received April 6, 2010; revised June 30, 2010

A. Thomas, School of Mathematics and Statistics F07, University of Sydney, Sydney NSW 2006, Australia

E-mail: anne.thomas@sydney.edu.au 